\title{
CLASSIFICATION OF PARTIALLY HYPERBOLIC DIFFEOMORPHISMS IN 3-MANIFOLDS WITH SOLVABLE FUNDAMENTAL GROUP
}

\author{
ANDY HAMMERLINDL AND RAFAEL POTRIE
}

\begin{abstract}
A classification of partially hyperbolic diffeomorphisms on 3-dimensional manifolds with (virtually) solvable fundamental group is obtained. If such a diffeomorphism does not admit a periodic attracting or repelling two-dimensional torus, it is dynamically coherent and leaf conjugate to a known algebraic example. This classification includes manifolds which support Anosov flows, and it confirms conjectures by Rodriguez Hertz-Rodriguez Hertz-Ures ( $\overline{\mathrm{RHRHU}_{2}}$ ) and Pujals ([BoW] $)$ in the specific case of solvable fundamental group.
\end{abstract}

Keywords: Partial hyperbolicity (pointwise), dynamical coherence, leaf conjugacy. MSC 2000: 37C05, 37C20, 37C25, 37C29, 37D30, 57R30.

\section{INTRODUCTION}

1.1. Partial hyperbolicity in dimension three. Partial hyperbolicity has been widely studied in recent years not only as a generalization of uniform hyperbolicity, but also to characterize robust dynamical behaviour in terms of geometric structures invariant under the tangent map. Partially hyperbolic diffeomorphisms play a central role in both the study of stable ergodicity and robust transitivity (see [BDV, Wi]).

The goal of this paper is to contribute to the classification problem of partially hyperbolic diffeomorphisms in dimension three. A diffeomorphism $f$ of a 3-manifold $M$ is partially hyperbolic if there is a splitting of the tangent bundle into three $D f$-invariant one-dimensional subbundles $T M=E^{s} \oplus E^{c} \oplus E^{u}$ such that $E^{s}$ is contracted by $D f, E^{u}$ is expanded by $D f$, and this dominates any expansion or contraction on $E^{c}$.

The classification can be stated informally as follows:

Main Theorem. Every partially hyperbolic diffeomorphism on a 3-manifold with (virtually) solvable fundamental group is, up to finite lifts and iterates, one of the following:

- a Derived-from-Anosov system,

- a skew-product,

- a deformation of a suspension Anosov flow, or

A.H. was partially supported by the Australian Research Council under Grant DP120104514. R.P. was partially supported by CSIC group 618, FCE-3-2011-1-6749 and the Palis-Balzan project. 
- a non-dynamically coherent system containing

a periodic torus tangent either to $E^{c} \oplus E^{u}$ or $E^{c} \oplus E^{s}$.

The next section gives precise statements both of the definition of partial hyperbolicity and of the four families in the classification.

It is necessary to consider finite iterates and lifts to a finite cover as otherwise the result would not hold (see [BoW]). In Appendix $\mathrm{A}$, we present a complete classification which takes into account all possible quotients and roots of the above models.

All four families in the Main Theorem are non-empty. For the first three, simple algebraic models exist. Constructing examples in the fourth family, however, requires much more work. The recent proof of the existence of such non-dynamically coherent examples, given in $\mathrm{RHRHU}_{2}$, came as a surprise to the partially hyperbolic community. Since a periodic torus must be an attractor if transverse to $E^{s}$ or a repeller if transverse to $E^{u}$, the existence of such objects can be ruled out under mild additional assumptions. For instance, there are no such tori if the partially hyperbolic system is transitive (or even chain recurrent) or has an invariant measure of full support. Appendix B further studies the dynamics of systems containing these periodic tori.

The proof of the Main Theorem builds on previous results such as $\mathrm{BBI}, \mathrm{BBI}_{2}, \mathrm{BI}$, $\left.\mathrm{RHRHU}_{1}, \mathrm{H}_{1}, \mathrm{H}_{2}, \mathrm{HP}, \mathrm{Par}, \mathrm{Pot}\right]$ and ideas developed in [BoW]. In fact, [HP] establishes the Main Theorem in the case of virtually nilpotent fundamental group.

The contribution of this paper is to treat for the first time partially hyperbolic diffeomorphisms on manifolds which support Anosov flows (namely 3-manifolds with sol geometry) without a priori assumptions on the existence of foliations tangent to the center direction. For these manifolds, no previous results on dynamical coherence were known, not even in the more restrictive case of absolute partial hyperbolicity.

We expect many of the ideas presented here to apply in more general contexts in order to complete the classification of partial hyperbolicity on all 3-manifolds. In fact, much of the reasoning here relies only on two crucial properties which hold in this case:

(i) The mapping class group of the manifold is finite; every diffeomorphism has an iterate which is isotopic to the identity.

(ii) Foliations of the manifold can be accurately described; if there are no torus leaves, no two leaves on the universal cover have bounded Hausdorff distance.

Using these two properties, we are able to show that all the leaves of certain branching foliations introduced in [BI] are fixed by the dynamics on the universal cover. This is already a big step in comparing such partially hyperbolic diffeomorphisms to Anosov flows as these flows always fix the leaves of their invariant weak foliations. This property of fixed leaves is used to classify Anosov and expansive flows on a variety of 3-manifolds 
(see $[\mathrm{Bru}]$ and the references therein), and such results might be generalized to the partially hyperbolic setting.

1.2. Precise definitions and statement of results. As this paper deals only with manifolds in dimension three, $M$ will always denote a compact connected 3-manifold without boundary. A diffeomorphism $f: M \rightarrow M$ is partially hyperbolic if there is $N>0$ and a $D f$-invariant continuous splitting $T M=E^{s} \oplus E^{c} \oplus E^{u}$ into one-dimensional subbundles such that for every $x \in M$ :

$$
\left\|\left.D f^{N}\right|_{E^{s}(x)}\right\|<\left\|\left.D f^{N}\right|_{E^{c}(x)}\right\|<\left\|\left.D f^{N}\right|_{E^{u}(x)}\right\| \quad \text { and }\left\|\left.D f^{N}\right|_{E^{s}(x)}\right\|<1<\left\|\left.D f^{N}\right|_{E^{u}(x)}\right\| .
$$

This definition is sometimes called strong partial hyperbolicity, as opposed to weak partially hyperbolicity, where there is only a splitting into two subbundles. (See [BDV, Appendix B] for more discussion.) There also exists an absolute version of partial hyperbolicity considered in Appendix B of this paper.

A foliation is a partition of $M$ into $C^{1}$-injectively immersed submanifolds tangent to a continuous distribution (see [C] ).

For all partially hyperbolic systems, the bundles $E^{s}$ and $E^{u}$ integrate into invariant stable and unstable foliations $\mathcal{W}^{s}$ and $\mathcal{W}^{u}$ (see [HPS]). The integrability of the other bundles does not hold in general. The system is dynamically coherent if there exist invariant foliations $\mathcal{W}^{c s}$ and $\mathcal{W}^{c u}$ tangent to $E^{c s}=E^{s} \oplus E^{c}$ and $E^{c u}=E^{c} \oplus E^{u}$ respectively. This automatically implies the existence of an invariant foliation $\mathcal{W}^{c}$ tangent to $E^{c}$. See $[\mathrm{BuW}]$ for more information on dynamical coherence and reasons for considering this definition.

As mentioned earlier, there are non-dynamically coherent examples which contain normally hyperbolic periodic two-dimensional tori. The discoverers of these examples conjectured that such tori are the unique obstruction to dynamical coherence in dimension three:

Conjecture (Rodriguez Hertz-Rodriguez Hertz-Ures (2009) RHRHU $\left._{2}\right]$ ). Let $f: M \rightarrow$ $M$ be a partially hyperbolic diffeomorphism such that there is no periodic two-dimensional torus tangent to $E^{c s}$ or $E^{c u}$. Then, $f$ is dynamically coherent.

We prove this conjecture in the case of virtually solvable fundamental group.

Theorem A. Let $f$ be a partially hyperbolic diffeomorphism of a 3-manifold $M$ such that $\pi_{1}(M)$ is virtually solvable. If there is no periodic torus tangent to $E^{c s}$ or $E^{c u}$, then there are unique invariant foliations tangent to $E^{c s}$ and $E^{c u}$. In particular, $f$ is dynamically coherent.

Once dynamical coherence is established, there is a natural way to classify partially hyperbolic diffeomorphisms from a topological point of view. This uses the notion of 
leaf conjugacy first introduced in [HPS] (see also [BoW]). Two partially hyperbolic diffeomorphisms $f, g: M \rightarrow M$ are leaf conjugate if they are both dynamically coherent and there is a homeomorphism $h: M \rightarrow M$ which maps every center leaf $L$ of $f$ to a center leaf $h(L)$ of $g$ and such that $h(f(L))=g(h(L))$.

In 2001, E. Pujals stated a conjecture (later formalized in [BoW]) that in dimension three all transitive partially hyperbolic diffeomorphisms belong to one of three already known classes.

Conjecture (Pujals (2001), Bonatti-Wilkinson (2004)). Let $f$ be a transitive partially hyperbolic diffeomorphism on a 3-manifold. Then, modulo finite lifts and iterates, $f$ is leaf conjugate to one of the following models:

- A linear Anosov diffeomorphism of $\mathbb{T}^{3}$.

- A skew product over a linear Anosov automorphism of $\mathbb{T}^{2}$.

- The time-one map of an Anosov flow.

Recently, Rodriguez Hertz, Rodriguez Hertz, and Ures stated a slightly different version of this conjecture where "transitive" is replaced by "dynamically coherent."

Our Main Theorem is then a proof of the conjecture in the case of virtually solvable fundamental group.

Main Theorem (Precise Statement). Let $f$ be a partially hyperbolic diffeomorphism on a 3-manifold with virtually solvable fundamental group. If there is no periodic torus tangent to $E^{c s}$ or $E^{c u}$, then, modulo finite lifts and iterates, $f$ is leaf conjugate to one of the following models:

- A linear Anosov diffeomorphism of $\mathbb{T}^{3}$.

- A skew product over a linear Anosov automorphism of $\mathbb{T}^{2}$.

- The time-one map of a suspension Anosov flow.

In the first case, the Anosov diffeomorphism $A: \mathbb{T}^{3} \rightarrow \mathbb{T}^{3}$ will have three distinct real eigenvalues and therefore be partially hyperbolic. In the second case, a skew product is a bundle map $f: M \rightarrow M$ where $M$ is a circle bundle over $\mathbb{T}^{2}$ defined by a continuous bundle projection $P: M \rightarrow \mathbb{T}^{2}$ and an Anosov base map $A: \mathbb{T}^{2} \rightarrow \mathbb{T}^{2}$ such that $P f=A P$. This family includes the standard skew products on $\mathbb{T}^{3}=\mathbb{T}^{2} \times \mathbb{T}^{1}$, as well as partially hyperbolic maps on other 3-dimensional nilmanifolds. See [HP] for further discussion on these two cases.

This paper mainly treats the third case. In this paper, a suspension manifold is taken to mean a manifold of the form $M_{A}=\mathbb{T}^{2} \times \mathbb{R} / \sim$ where $(A x, t) \sim(x, t+1)$ and $A: \mathbb{T}^{2} \rightarrow \mathbb{T}^{2}$ is a hyperbolic toral automorphism. A suspension Anosov flow is a flow $\varphi_{t}(x, s)=(x, s+t)$ on a suspension manifold $M_{A}$. 
Previous classification results used either the fact that the action in homology was partially hyperbolic $\left(\left[\mathrm{BI}, \mathrm{H}_{1}, \mathrm{H}_{2}, \mathrm{HP}\right]\right)$ or that many center leaves were fixed by $f([\mathrm{BoW}])$. In fact, based on many previous results, it was proved in [HP] that the previous conjectures hold under the assumption that the fundamental group of $M$ is virtually nilpotent (which is equivalent to having polynomial growth of volume).

In this paper, we treat a family of manifolds which admit Anosov flows and show that every partially hyperbolic system on these manifolds is comparable to a flow.

Theorem B. Let $f: M \rightarrow M$ be a partially hyperbolic dynamically coherent diffeomorphism on a manifold $M$ such that $\pi_{1}(M)$ is virtually solvable but not virtually nilpotent. Then, there exists a finite iterate of $f$ which is leaf conjugate to the time-one map of a suspension Anosov flow.

To end this introduction, we present an algebraic version of the Main Theorem which follows directly from the results presented in Appendix A. For a Lie group $G$, an affine map is a diffeomorphism of the form $G \rightarrow G, g \mapsto \Phi(g) \cdot g_{0}$ where $\Phi: G \rightarrow G$ is a Lie group automorphism and $g_{0} \in G$. Let $\operatorname{Aff}(G)$ denote the set of affine maps on $G$.

Theorem. If $f$ is a partially hyperbolic, dynamically coherent diffeomorphism of a 3manifold $M$ with (virtually) solvable fundamental group, then there are $G$, a solvable Lie group, $\Gamma<\operatorname{Aff}(G)$, and $\alpha \in \operatorname{Aff}(G)$ such that $G / \Gamma$ is a manifold diffeomorphic to $M, \alpha$ descends to a partially hyperbolic map $\alpha_{0}: G / \Gamma \rightarrow G / \Gamma$ and $f$ and $\alpha_{0}$ are leaf conjugate.

1.3. Main steps of the proof and organization of the paper. The Main Theorem has already been proven in the case of a virtually nilpotent fundamental group [HP]. This paper therefore proves the theorem only in the case that the group is virtually solvable and not virtually nilpotent. This breaks into five major steps.

Proposition 1.1. If $f$ is a partially hyperbolic diffeomorphism of a 3-manifold $M$ with a fundamental group which is virtually solvable and not virtually nilpotent, then there is a finite normal covering of $M$ by a suspension manifold $M_{A}$.

Proposition 1.2. If $M$ is finitely covered by a suspension manifold and $f: M \rightarrow M$ is partially hyperbolic, then $M$ is diffeomorphic to a suspension manifold.

Proposition 1.3. For any homeomorphism $f$ of a suspension manifold $M_{A}$, there is $n \geq 1$ such that $f^{n}$ is homotopic to the identity.

Proposition 1.4. If $f$ is a partially hyperbolic diffeomorphism on a suspension manifold $M_{A}$ and there is no periodic torus tangent to $E^{c u}$ or $E^{c s}$, then there are unique invariant foliations tangent to $E^{c u}$ and $E^{c s}$.

Proposition 1.5. If $f$ is a partially hyperbolic diffeomorphism on a suspension manifold $M_{A}$ such that $f$ is dynamically coherent and homotopic to the identity, then $f$ is leaf conjugate to the time-one map of a suspension Anosov flow. 
Together these propositions also imply Theorems A and B. (In the case of Theorem A, one must also refer to $[\mathrm{HP}$ ] for the virtually nilpotent case.) Thus, the remaining goal of this paper is to prove each of the five propositions.

Section 2 proves Proposition 1.1 based on results given in $[\mathrm{Par}]$. The section also reduces Proposition 1.4 to an equivalent result (Lemma 2.1) which is easier to prove.

Fundamental to the proofs of Propositions 1.4 and 1.5 are the branching foliations $\mathcal{F}^{c s}$ and $\mathcal{F}^{c u}$ developed by Burago and Ivanov [BI]. Section 3 introduces these objects and their properties.

Section 4 details the geometry of the suspension manifold $M_{A}$, its universal cover $\tilde{M}_{A}$, and defines two model foliations $\mathcal{A}^{c s}$ and $\mathcal{A}^{c u}$ on this cover.

Section 5 compares the branching foliations to the model foliations showing that each leaf of $\mathcal{F}^{c s}$ is a finite distance from a leaf of $\mathcal{A}^{c s}$ and vice versa, and that the same holds for $\mathcal{F}^{c u}$ and $\mathcal{A}^{c u}$.

Section 6 shows dynamical coherence, completing the proof of Proposition 1.4 .

Section 7 establishes the leaf conjugacy, Proposition 1.5.

Appendix $\mathrm{A}$ proves Propositions 1.2 and 1.3 and thus finishes the proof of the Main Theorem. This appendix also gives an algebraic classification of partially hyperbolic diffeomorphisms on suspension manifolds which are not homotopic to the identity and classifies the possible quotients of partially hyperbolic diffeomorphisms on the 3-torus and other 3-nilmanifolds.

Appendix $\mathrm{B}$ considers the case where the branching foliation $\mathcal{F}^{c s}$ or $\mathcal{F}^{c u}$ has a torus leaf. This implies the existence of a periodic torus, and the appendix rules out such tori for absolutely partially hyperbolic systems.

Acknowledgements: We have benefited from discussions with C. Bonatti, J. Brum, S. Crovisier, E. Pujals, J.A.G. Roberts, M. Sambarino, and A. Wilkinson.

\section{REDUCtion to THE CASE OF A SUSPENSION MANIFOLD}

We start by proving Proposition 1.1 .

Proof of Proposition 1.1. Parwani showed that if $f$ is partially hyperbolic on a 3-manifold $M$ with virtually solvable fundamental group, then $M$ is finitely covered by a torus bundle over a circle [Par, Theorem 1.10]. That is, $M$ is finitely covered by a suspension manifold $M_{A}$ with the caveat that the toral automorphism $A$ is not necessarily hyperbolic. Then, the fundamental group of $M_{A}$ verifies $\pi_{1}\left(M_{A}\right) \cong \mathbb{Z}^{2} \rtimes_{A} \mathbb{Z}$, and if $A$ is not hyperbolic, a direct computation shows that the group must be nilpotent implying that the fundamental group of $M$ is virtually nilpotent. As this is not possible by hypothesis, $A$ must be hyperbolic. 
Remark. The exact statement in [Par, Corollary 1.11] requires that the fundamental group has a normal finite-index solvable subgroup $K \triangleleft \pi_{1}(M)$, instead of just a finiteindex solvable subgroup $H<\pi_{1}(M)$. These two conditions are, in fact, equivalent. Any finite-index subgroup $H<G$ contains a normal finite-index subgroup $K \triangleleft G$. Indeed, there is a natural homomorphism $g \mapsto(a H \mapsto g a H)$ from $G$ to the (finite) group of permutations of the cosets of $H$, and we may take $K$ to be the kernel. If $H$ is solvable, the subgroup $K<H$ is also solvable.

Section 6 gives the proof of the following lemma which adds two additional assumptions to Proposition 1.4 .

Lemma 2.1. If $f$ is a partially hyperbolic diffeomorphism on a suspension manifold $M_{A}$ such that

- there is no $f$-periodic torus tangent to $E^{c u}$ or $E^{c s}$,

- $E^{u}, E^{c}, E^{s}$ are oriented and $D f$ preserves these orientations, and

- $f$ is homotopic to the identity,

then there are unique $f$-invariant foliations tangent to $E^{c u}$ and $E^{c s}$.

To see how Proposition 1.4 follows from this lemma, we need two results involving finite covers.

Proposition 2.2. If $f: M \rightarrow M$ is a homeomorphism of a manifold and $p: N \rightarrow M$ is a finite cover, then some iterate of $f$ lifts to $N$. To be precise, there is $n \geq 1$ and $g: N \rightarrow N$ such that $p \circ g=f^{n} \circ p$.

Proof. In order to lift a map $h: M \rightarrow M$ to $N$ by the cover $p$ one must verify that $h_{*} p_{*}\left(\pi_{1}(N)\right) \subset p_{*}\left(\pi_{1}(N)\right)$. Since $p$ is a finite cover, the subgroup $p_{*}\left(\pi_{1}(N)\right)$ has finite index in $\pi_{1}(M)$. Let $k \geq 1$ be this index.

Given a group $G$ and $k \geq 1$, there are finitely many subgroups of $G$ of index $k$ [LS, Theorem IV.4.7]. Therefore, there exists $n \geq 1$ such that $\left(f^{n}\right)_{*}\left(p_{*}\left(\pi_{1}(N)\right)\right)=p_{*}\left(\pi_{1}(N)\right)$ and one can lift $f^{n}$ to $N$ as desired.

Proposition 2.3. Suppose

- $f: M \rightarrow M$ is a diffeomorphism,

- $E_{M} \subset T M$ is a Df-invariant subbundle,

- $p: N \rightarrow M$ is a finite normal cover,

- $E_{N} \subset T N$ is the lift of $E_{M}$, and

- $g: N \rightarrow N$ is a diffeomorphism such that $p \circ g=f^{n} \circ p$ for some $n \geq 1$.

If there is a unique g-invariant foliation $\mathcal{W}_{N}$ tangent to $E_{N}$, then there is also a unique $f$-invariant foliation $\mathcal{W}_{M}$ tangent to $E_{M}$. 
Proof. First, suppose there is a unique $f^{n}$-invariant foliation $\mathcal{W}$ tangent to $E_{M}$. Then, $f(\mathcal{W})$ is also an $f^{n}$-invariant foliation and by uniqueness $f(\mathcal{W})=\mathcal{W}$. That is, $\mathcal{W}$ is an $f$-invariant foliation and since any $f$-invariant foliation is also $f^{n}$-invariant, it is the unique one. This shows that we may freely replace $f$ and $g$ by their iterates in the rest of the proof.

Let $\Gamma$ be the finite group of deck transformations $\gamma: N \rightarrow N$ associated to the cover $p: N \rightarrow M$. Note that $\gamma \mapsto g^{-1} \gamma g$ is an automorphism of $\Gamma$. As $\Gamma$ is finite, there is $m$ such that $\gamma=g^{-m} \circ \gamma \circ g^{m}$ for all $m$. By replacing $f$ and $g$ by iterates, assume that $m=1$ and $p \circ g=f \circ p$.

If $\mathcal{W}_{N}$ is the unique $g$-invariant foliation tangent to $E_{N}, \gamma \circ g=g \circ \gamma$ implies that $\gamma\left(\mathcal{W}_{N}\right)$ is also $g$-invariant, and as $E_{N}$ was lifted from a subbundle of $T M, \gamma\left(\mathcal{W}_{N}\right)$ is also tangent to $E_{N}$. By uniqueness, $\gamma\left(\mathcal{W}_{N}\right)=\mathcal{W}_{N}$ for all $\gamma \in \Gamma$, so $\mathcal{W}_{N}$ projects down to a foliation $\mathcal{W}_{M}$ on $M$. As $p \circ g=f \circ p$ and locally $p$ is a diffeomorphism, it follows that $\mathcal{W}_{M}$ is $f$-invariant. Uniqueness of $\mathcal{W}_{N}$ implies uniqueness of $\mathcal{W}_{M}$.

We now prove Proposition 1.4 assuming Lemma 2.1 and Proposition 1.3 ,

Proof of Proposition 1.4. There is a finite cover $p: M_{1} \rightarrow M_{A}$ such that when lifted to $M_{1}$ the bundles $E^{u}, E^{c}, E^{s}$ are orientable. One can show that $M_{1}$ is again a suspension manifold. By Lemma 2.2, there is a map $f_{1}: M_{1} \rightarrow M_{1}$ such that $p \circ f_{1}=f^{k} \circ p$. By Proposition 1.3, there is $n$ such that $f_{1}^{n}$ is homotopic to the identity. Then $g:=f_{1}^{2 n}$ is also homotopic to the identity and preserves the orientations of the bundles. Note that if there is a $g$-periodic 2-torus tangent to $E^{c u}$ or $E^{c s}$, it projects to an $f$-periodic surface tangent to $E^{c u}$ or $E^{c s}$ which must again be a torus. As such a torus is ruled out by assumption, the conditions of Lemma 2.1 hold and there are unique $g$-invariant foliations on $M_{1}$ tangent to $E^{c u}$ and $E^{c s}$. Since $p \circ g=f^{2 n k} \circ p$, Lemma 2.3 implies that there are unique $f$-invariant foliations on $M_{A}$ tangent to $E^{c u}$ and $E^{c s}$.

\section{BRANCHING FOLIATIONS}

The starting point for many recent results in partial hyperbolicity is the masterful construction by Burago and Ivanov of branching foliations associated to the dynamics [BI]. Using Novikov's compact leaf theorem and other deep properties in the foliation theory of 3-manifolds, Burago and Ivanov proved a number of results which may be stated as follows.

Theorem 3.1 (Burago-Ivanov [BI]). Let $f_{0}: M \rightarrow M$ be a partially hyperbolic diffeomorphism on a 3-manifold such that $E^{u}, E^{c}$, and $E^{s}$ are orientable and the derivative $D f_{0}$ preserves these orientations. Let $f: \tilde{M} \rightarrow \tilde{M}$ be a lift of $f_{0}$ to the universal cover.

Then, there is a collection $\mathcal{F}^{c s}$ of properly embedded planes in $\tilde{M}$ such that for every $L \in \mathcal{F}^{c s}$ : 
(1) $L$ is tangent to $E^{c s}$,

(2) $f(L) \in \mathcal{F}^{c s}$,

(3) $\gamma(L) \in \mathcal{F}^{c s}$ for all deck transformations $\gamma \in \pi_{1}(M)$,

(4) $L$ cuts $\tilde{M}$ into two closed half-spaces $L^{+}$and $L^{-}$, and

(5) every $L^{\prime} \in \mathcal{F}^{c s}$ lies either in $L^{+}$or $L^{-}$(or both if $L=L^{\prime}$ ).

Finally, for every $x \in \tilde{M}$ there is at least one $L \in \mathcal{F}^{c s}$ such that $x \in L$.

An analogous collection $\mathcal{F}^{c u}$ also exists. For the purposes of this paper, a branching foliation will be a collection as in the statement of this theorem. The planes of the collection will be called leaves of the branching foliation in analogy with true foliations. Item (5) of the list is equivalent to saying that leaves of the branching foliation do not topologically cross.

Assumption 3.2. For the rest of this section, assume $f_{0}, f, M, \tilde{M}$, and $\mathcal{F}^{c s}$ are as in Theorem 3.1.

Proposition 3.3. Each leaf of $\mathcal{F}^{c s}$ intersects an unstable leaf in at most one point.

Proof. On the universal cover $\tilde{M}$, one may choose an orientation of $E^{u}$ such that for a leaf $L \in \mathcal{F}^{c s}$ and at all points $x \in L$ the orientation of $E^{u}(x)$ is away from $L^{-}$and into $L^{+}$. Therefore, any oriented path tangent to $E^{u}$ passes from $L^{-}$into $L^{+}$when intersecting $L$, and a path can do this at most once.

The following proposition, which follows from [BoW, Remark 1.16], will be used to establish dynamical coherence.

Proposition 3.4. If every $x \in \tilde{M}$ is contained in exactly one leaf of $\mathcal{F}^{c s}$, then $\mathcal{F}^{c s}$ is a true foliation.

Even when $\mathcal{F}^{c s}$ is not a true foliation, it is close to a true foliation in a certain sense. To state this precisely, we introduce a few more definitions.

In a metric space $X$ where $d(\cdot, \cdot)$ is the distance function and $Y$ and $Z$ are non-empty subsets, define $\operatorname{dist}(x, Z):=\inf _{z \in Z} d(x, z)$ and Hausdorff distance as

$$
d_{H}(Y, Z):=\max \left\{\sup _{y \in Y} \operatorname{dist}(y, Z), \sup _{z \in Z} \operatorname{dist}(z, Y)\right\}
$$

The Hausdorff distance may be infinite in general.

Suppose $\mathcal{F}_{1}$ and $\mathcal{F}_{2}$ are collections of subsets of a metric space $X$. Then, $\mathcal{F}_{1}$ and $\mathcal{F}_{2}$ are almost parallel if there is $R>0$ such that

- for each $L_{1} \in \mathcal{F}_{1}$, there is $L_{2} \in \mathcal{F}_{2}$ with $d_{H}\left(L_{1}, L_{2}\right)<R$, and

- for each $L_{2} \in \mathcal{F}_{2}$, there is $L_{1} \in \mathcal{F}_{1}$ with $d_{H}\left(L_{2}, L_{1}\right)<R$. 
In our case, these collections will either be foliations or branching foliations and $X$ will be the universal cover of a manifold.

Proposition 3.5. The branching foliation $\mathcal{F}^{c s}$ is almost parallel to a foliation $\mathcal{F}$ of $\tilde{M}$ by planes and this foliation quotients down to a foliation $\mathcal{F}_{0}$ on $M$. Moreover, $\mathcal{F}_{0}$ contains a compact surface $S$ if and only if there is $L \in \mathcal{F}^{c s}$ which quotients down to a compact surface on $M$ homeomorphic to $S$.

This follows as a consequence of [BI, Theorem 7.2].

The existence of a compact surface coming from the branching foliations has strong dynamical consequences.

Proposition 3.6. If $\mathcal{F}^{c s}$ has a leaf $L$ which projects to a compact surface on $M$, then there is a normally repelling $f_{0}$-periodic torus tangent to $E^{c s}$.

This is proved in Appendix B, where partially hyperbolic systems with such tori are studied in further detail.

In the specific case of 3-manifolds with solvable fundamental group, the foliations without compact leaves have been classified. This was done by Plante for $C^{2}$ foliations where the classification is up to equivalence [Pl]. For the foliations considered in this paper, the classification only holds up to the notion of two foliations being almost parallel when lifted to the universal cover [HP]. In the case of suspension manifolds $M_{A}$, any such foliation, after lifting, is almost parallel to one of two model foliations, $\mathcal{A}^{c s}$ or $\mathcal{A}^{c u}$, which will be defined in the next section.

Proposition 3.7. If $\mathcal{F}_{0}$ is a foliation of $M_{A}$ without compact leaves, then the lifted foliation $\mathcal{F}$ on $\tilde{M}_{A}$ is almost parallel either to $\mathcal{A}^{c s}$ or $\mathcal{A}^{c u}$.

This is a restatement of $[\mathrm{HP}$, Theorem B.6].

\section{Solvmanifolds And MOdel FOliations}

Suppose $A$ is a hyperbolic $2 \times 2$ integer matrix with integer entries and determinant plus-minus one. Then, there is $\lambda>1$ such that $A$ has an unstable eigenspace in $\mathbb{R}^{2}$ with associated eigenvalue $\pm \lambda$, and a stable eigenspace with associated eigenvalue $\pm \lambda^{-1}$.

Consider a manifold $\tilde{M}_{A}$ diffeomorphic to $\mathbb{R}^{2} \times \mathbb{R}$, but with a non-Euclidean geometry to be defined shortly. Each point in $\tilde{M}_{A}$ can be written as $(v, t)$ with $v \in \mathbb{R}^{2}$ and $t \in \mathbb{R}$. Define foliations $\mathcal{A}^{c s}$ and $\mathcal{A}^{c u}$ of dimension two by:

- $\left(v_{1}, t_{1}\right) \in \mathcal{A}^{c s}\left(\left(v_{2}, t_{2}\right)\right)$ if $v_{1}-v_{2}$ is in the stable eigenspace of $A$,

- $\left(v_{1}, t_{1}\right) \in \mathcal{A}^{c u}\left(\left(v_{2}, t_{2}\right)\right)$ if $v_{1}-v_{2}$ is in the unstable eigenspace of $A$,

Define foliations $\mathcal{A}^{s}$ and $\mathcal{A}^{u}$ of dimension one by: 


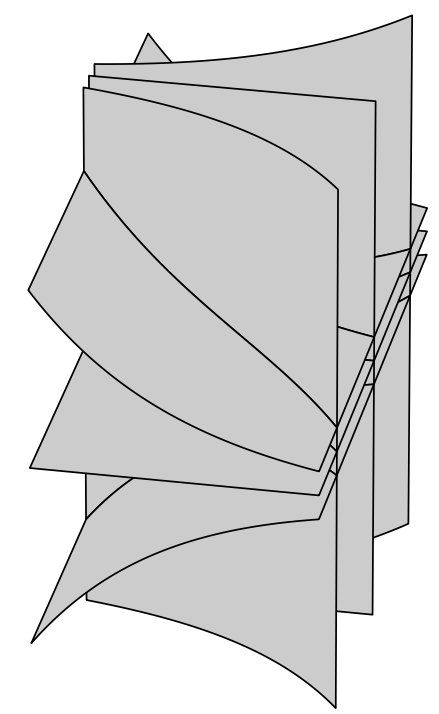

Figure 1. A depiction of the model foliations $\mathcal{A}^{c s}$ and $\mathcal{A}^{c u}$. The planes separate apart in the left direction for one foliation and in the right direction for the other. Note that in the coordinate system for $\tilde{M}_{A} \approx \mathbb{R}^{3}$ introduced in Section 4, the foliations consist of geometric planes and it is the Riemannian metric which changes in $t$.

- $\left(v_{1}, t_{1}\right) \in \mathcal{A}^{s}\left(\left(v_{2}, t_{2}\right)\right)$ if $t_{1}=t_{2}$ and $\left(v_{1}, t_{1}\right) \in \mathcal{A}^{c s}\left(\left(v_{2}, t_{2}\right)\right)$,

- $\left(v_{1}, t_{1}\right) \in \mathcal{A}^{u}\left(\left(v_{2}, t_{2}\right)\right)$ if $t_{1}=t_{2}$ and $\left(v_{1}, t_{1}\right) \in \mathcal{A}^{c u}\left(\left(v_{2}, t_{2}\right)\right)$.

Define a flow $\varphi: \tilde{M}_{A} \times \mathbb{R} \rightarrow \tilde{M}_{A}$ by $\varphi_{t}((v, s))=(v, s+t)$ and let $\mathcal{A}^{c}$ be the foliation consisting of orbits of the flow. Now, define a unique Riemannian metric on $\tilde{M}_{A}$ by requiring the following properties:

- at every point in $\tilde{M}_{A}$, an orthogonal basis of the tangent space can be formed by vectors in $\mathcal{A}^{s}, \mathcal{A}^{c}$, and $\mathcal{A}^{u}$,

- $\varphi$ is a unit speed flow,

- if $\alpha$ is a simple curve in a leaf of $\mathcal{A}^{u}$ with endpoints $\left(v_{1}, t\right)$ and $\left(v_{2}, t\right)$, then length $(\alpha)=\lambda^{t}\left\|v_{1}-v_{2}\right\|$, and

- if $\alpha$ is a simple curve in a leaf of $\mathcal{A}^{s}$ with endpoints $\left(v_{1}, t\right)$ and $\left(v_{2}, t\right)$, then length $(\alpha)=\lambda^{-t}\left\|v_{1}-v_{2}\right\|$.

In the last two items above, $\left\|v_{1}-v_{2}\right\|$ denotes the Euclidean distance in $\mathbb{R}^{2}$. Define functions $\gamma_{i}: \tilde{M}_{A} \rightarrow \tilde{M}_{A}$ by

$$
\begin{aligned}
\gamma_{1}((v, t)) & =(v+(1,0), t), \\
\gamma_{2}((v, t)) & =(v+(0,1), t), \\
\gamma_{3}((A v, t)) & =(v, t+1) .
\end{aligned}
$$


One can verify that these three functions are isometries which generate a solvable group $G \subset \operatorname{Isom}\left(\tilde{M}_{A}\right)$ and that the quotient $\tilde{M}_{A} / G$ is exactly the compact manifold $M_{A}$ defined earlier. Further, the flow $\varphi$ quotients to an Anosov flow on $M_{A}$ and the foliations $\mathcal{A}^{*}$ quotient down to invariant foliations associated to the flow. Define $G_{0}=\left\langle\gamma_{1}, \gamma_{2}\right\rangle$.

Define a function $p_{1}: \tilde{M}_{A} \rightarrow \mathbb{R},(v, t) \mapsto t$.

Lemma 4.1. For all $\gamma \in G$, one has $p_{1}(x)-p_{1}(y)=p_{1}(\gamma(x))-p_{1}(\gamma(y))$. Further, $p_{1} \circ \gamma=p_{1}$ exactly when $\gamma \in G_{0}$.

Proof. This follows from the definitions of $p_{1}$ and $\gamma_{i}(i=1,2,3)$.

Lemma 4.2. For any $x, y \in \tilde{M}_{A}$, one has $\left|p_{1}(x)-p_{1}(y)\right| \leq d(x, y)$.

Proof. Consider a curve $\alpha:[a, b] \rightarrow \tilde{M}_{A}$ from $x$ to $y$ parameterized by arc length. For each point $\alpha(t)$, there is an orthonormal basis $\left\{v^{u}, v^{c}, v^{s}\right\}$ of the tangent space such that the exterior derivative $d p_{1}$ satisfies $d p_{1}\left(v^{u}\right)=0, d p_{1}\left(v^{c}\right)=1$, and $d p_{1}\left(v^{s}\right)=0$. This shows that

and therefore $\left|p_{1}(y)-p_{1}(x)\right| \leq b-a$.

$$
\left|\frac{d}{d t} p_{1}(\alpha(t))\right| \leq 1
$$

For any $x, y \in \tilde{M}_{A}$, there is a unique point of intersection

$$
[x, y]:=\mathcal{A}^{u}(x) \cap \mathcal{A}^{c s}(y) .
$$

This point depends continuously on $x$ and $y$ and since the foliations are invariant under $G$,

$$
[\gamma(x), \gamma(y)]=\gamma([x, y])
$$

for all $\gamma \in G$. Define $\operatorname{dist}_{u}\left(x, \mathcal{A}^{c s}(y)\right)$ as the length of the unstable segment between $x$ and $[x, y]$. Note that

$$
\operatorname{dist}_{u}\left(\varphi_{t}(x), \mathcal{A}^{c s}(y)\right)=\lambda^{t} \operatorname{dist}_{u}\left(x, \mathcal{A}^{c s}(y)\right)
$$

for all $x, y$, and $t$.

Lemma 4.3. For $R>0$ there is $R_{1}>0$ such that

$$
\operatorname{dist}(x, \mathcal{L})<R \Rightarrow \operatorname{dist}_{u}(x, \mathcal{L})<R_{1}
$$

for all $x \in \tilde{M}_{A}$ and $\mathcal{L} \in \mathcal{A}^{c s}$.

Proof. Define

$$
\Delta=\left\{(x, y) \in \tilde{M}_{A} \times \tilde{M}_{A}: d(x, y) \leq R\right\} .
$$

It is enough to show that $D: \Delta \rightarrow \mathbb{R}$ defined by $D(x, y)=\operatorname{dist}_{u}\left(x, \mathcal{A}^{c s}(y)\right)$ is bounded. Define an action of $G$ on $\Delta$ by $\gamma \cdot(x, y)=(\gamma(x), \gamma(y))$ and note that $D$ quotients to a continuous function $D_{G}: \Delta / G \rightarrow \mathbb{R}$. As $\Delta / G$ is compact, both $D_{G}$ and $D$ are bounded. 
Lemma 4.4. If $\left\{x_{k}\right\},\left\{x_{k}^{\prime}\right\}$ are sequences in leaves $\mathcal{L}, \mathcal{L}^{\prime} \in \mathcal{A}^{\text {cs }}$ such that $\sup d\left(x_{k}, x_{k}^{\prime}\right)<\infty$ and $\lim _{k \rightarrow \infty} p_{1}\left(x_{k}\right)=+\infty$, then $\mathcal{L}=\mathcal{L}^{\prime}$.

Proof. By the last lemma, there is $R_{1}$ such that $\operatorname{dist}_{u}\left(x_{k}, \mathcal{L}^{\prime}\right)<R_{1}$ for all $k$. Let $t_{k}=$ $-p_{1}\left(x_{k}\right)$. Then

$$
\lim _{k \rightarrow \infty} \operatorname{dist}_{u}\left(\varphi_{t_{k}}\left(x_{k}\right), \mathcal{L}^{\prime}\right) \leq \lim _{k \rightarrow \infty} \lambda^{t_{k}} R_{1}=0
$$

This shows that there are points in $\mathcal{L} \cap p_{1}^{-1}(0)$ which come arbitrarily close to $\mathcal{L}^{\prime} \cap p_{1}^{-1}(0)$. As the definition of $\mathcal{A}^{c s}$ implies that its leaves intersect the plane $p_{1}^{-1}(0)$ in parallel lines, the result follows.

Corollary 4.5. If $\mathcal{L}, \mathcal{L}^{\prime} \in \mathcal{A}^{c s}$ are distinct, then $d_{H}\left(\mathcal{L}, \mathcal{L}^{\prime}\right)=\infty$.

A leaf of $\mathcal{A}^{c s}$ splits $\tilde{M}_{A}$ into two pieces. We will also need that an arbitrarily large neighbourhood of a leaf splits $\tilde{M}_{A}$ into two pieces.

Lemma 4.6. For any $R>0$ and $\mathcal{L} \in \mathcal{A}^{c s}$, there is a partition of $\tilde{M}_{A}$ into three pieces $X, Y, Z$ such that

- $X$ and $Z$ are open and connected,

- $d_{H}(Y, \mathcal{L})<\infty$, and

- $\operatorname{dist}(y, \mathcal{L})<R$ implies $y \in Y$.

Proof. Let $Y=\left\{y \in \tilde{M}_{A}: \operatorname{dist}_{u}(y, \mathcal{L}) \leq R_{1}\right\}$ where $R_{1}$ is given by Lemma 4.3, Let $X, Z$ be the two connected components of $\tilde{M}_{A} \backslash Y$. It is straightforward to verify the desired properties.

Lemma 4.7. For any $n>0$ the manifold with boundary defined by

$$
\tilde{V}_{n}:=\left\{x \in \tilde{M}_{A}:\left|p_{1}(x)\right| \leq n\right\}
$$

has polynomial growth of volume. That is, if $B_{R}(x)$ consists of all endpoints of paths in $\tilde{V}_{n}$ starting at $x$ and of length at most $R$, then the volume of $B_{R}(x)$ grows polynomially in $R$.

Proof. The deck transformations in $G_{0}$ map $\tilde{V}_{n}$ to itself and the quotient $\hat{V}_{n}:=\tilde{V}_{n} / G_{0}$ is compact and is therefore a finite volume manifold with boundary. The result then follows from the fact that $G_{0} \cong \mathbb{Z}^{2}$ has polynomial growth.

Lemma 4.8. If $X$ is a measurable subset of $\tilde{M}_{A}$ such that $X \cap \gamma(X)=\varnothing$ for all $\gamma \in G_{0}$ then the volume of

$$
X_{n}:=\left\{x \in X:\left|p_{1}(x)\right|<n\right\}
$$

grows linearly in $n$. 
Proof. Using that the isometry $\gamma_{3} \in G$ is volume preserving, it is not hard to show that the volume of $\hat{V}_{n}$ (as defined in the proof of the last lemma) is exactly $n$ times the volume of $\hat{V}_{1}$. Each $X_{n} \subset \tilde{V}_{n}$ projects to a set $\hat{X}_{n} \subset \hat{V}_{n}$. As this projection is one-to-one, the volumes of $X_{n}$ and $\hat{X}_{n}$ are the same. The result follows.

\section{Comparing Foliations}

In this section, suppose $f_{0}$ is a partially hyperbolic diffeomorphism which satisfies the hypotheses of Lemma 2.1. In particular, since it is homotopic to the identity, $f_{0}$ lifts to a diffeomorphism $f: \tilde{M}_{A} \rightarrow \tilde{M}_{A}$ which is a finite distance from the identity.

In this section, the only dynamical system we consider is $f$. We do not consider the flow $\varphi$ used in the last section. Thus, the words stable, unstable, and center here refer only to $f$.

As the hypotheses of Theorem 3.1 are satisfied, there are branching foliations $\mathcal{F}^{c s}$ and $\mathcal{F}^{c u}$. By Propositions [3.5, 3.6, and 3.7, the branching foliation $\mathcal{F}^{c s}$ is almost parallel to either $\mathcal{A}^{c s}$ or $\mathcal{A}^{c u}$, defined in Section 4, Up to replacing $A$ with $A^{-1}$, we may freely assume the first.

Assumption 5.1. $\mathcal{F}^{c s}$ is almost parallel to $\mathcal{A}^{c s}$ and throughout the rest of the paper $R>0$ is the associated constant in the definition.

From this assumption it will follow that $\mathcal{F}^{c u}$ is almost parallel to $\mathcal{A}^{c u}$. However, the proof of this is surprisingly involved and will occupy the rest of this section. We must first prove a number of properties relating $\mathcal{F}^{c s}$ to $\mathcal{A}^{c s}$.

Lemma 5.2. If $L \in \mathcal{F}^{c s}, \mathcal{L} \in \mathcal{A}^{c s}$, and $d_{H}(L, \mathcal{L})<\infty$, then

(1) $\mathcal{L}$ is the unique leaf in $\mathcal{A}^{\text {cs }}$, such that $d_{H}(L, \mathcal{L})<\infty$,

(2) $d_{H}(L, \mathcal{L})<R$,

(3) $d_{H}\left(f^{k}(L), \mathcal{L}\right)<R$ for all $k \in \mathbb{Z}$, and

(4) $d_{H}(\gamma(L), \gamma(\mathcal{L}))<R$ for all $\gamma \in G$.

Proof. The first item follows from Corollary 4.5 and the fact that Hausdorff distance satisfies the triangle inequality. The second item follows from the first item and the definition of almost parallel. The third item then follows from the fact that $f$ is a bounded distance from the identity and therefore $d_{H}(f(X), X)<\infty$ for any subset $X \subset \tilde{M}_{A}$. The fourth item holds because $\gamma \in G$ is an isometry which preserves both $\mathcal{F}^{c s}$ and $\mathcal{A}^{c s}$.

Lemma 5.2 shows there is a canonical function from leaves of $\mathcal{F}^{c s}$ to leaves of $\mathcal{A}^{c s}$. This function is in fact a bijection. 


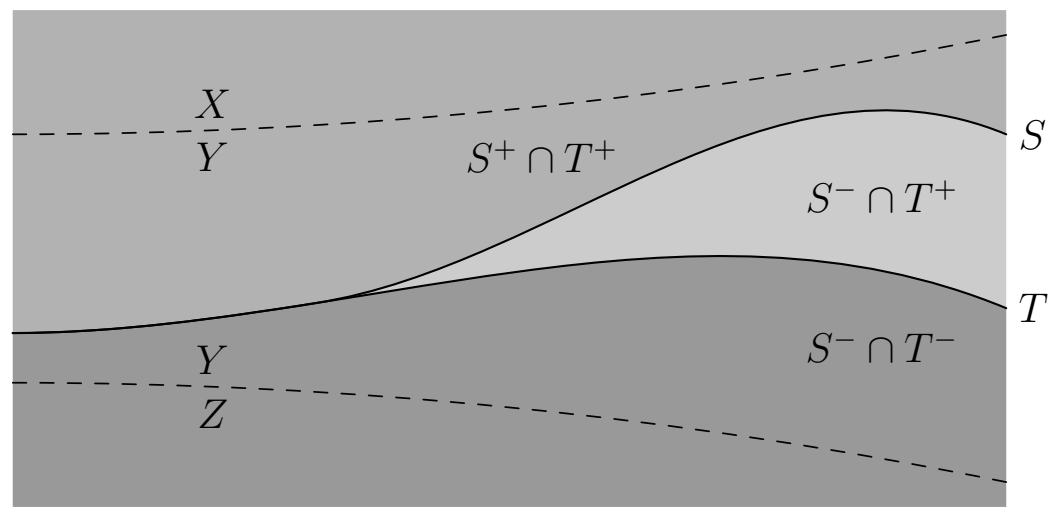

FiguRE 2. A depiction of the half-spaces in the proof of Lemma 5.4.

Lemma 5.3. If $L \in \mathcal{F}^{c s}, \mathcal{L} \in \mathcal{A}^{c s}$, and $d_{H}(L, \mathcal{L})<\infty$, then $L$ is the unique leaf in $\mathcal{F}^{c s}$ such that $d_{H}(L, \mathcal{L})<\infty$,

To prove this, we first provide two sublemmas.

Lemma 5.4. If for $\mathcal{L} \in \mathcal{A}^{\text {cs }}$ the union

$$
\Lambda=\bigcup\left\{L \in \mathcal{F}^{c s}: d_{H}(L, \mathcal{L})<\infty\right\}
$$

contains two distinct leaves of $\mathcal{F}^{c s}$, then this union has non-empty interior.

Proof. Let $X, Y, Z$ be as in Lemma 4.6. Note by Lemma 5.2 that if $L \in \mathcal{F}^{c s}$ is contained in $Y$, then $L \subset \Lambda$.

Suppose $S, T \in \mathcal{F}^{c s}$ are distinct leaves, both contained in $\Lambda$. As $S$ is a properly embedded surface, it splits $\tilde{M}_{A}$ into two closed half-spaces $S^{+}$and $S^{-}$such that

$$
\partial S^{+}=\partial S^{-}=S^{+} \cap S^{-}=S \quad \text { and } \quad \operatorname{int}\left(S^{+}\right) \cup \operatorname{int}\left(S^{-}\right)=\tilde{M}_{A} \backslash S .
$$

Since leaves of the branching foliation $\mathcal{F}^{c s}$ do not topologically cross, every leaf which intersects the interior of $S^{+}$is contained in $S^{+}$and similarly for $S^{-}$.

Since $S \subset Y, X$ must lie entirely in $S^{+}$or $S^{-}$. Assume $X \subset S^{+}$. If $Z$ is also a subset of $S^{+}$, then $S^{-}$would be contained in $Y$ and any leaf of $\mathcal{F}^{c s}$ which intersected the interior of $S^{-}$would be in $\Lambda$. As $S^{-}$has non-empty interior, this would be enough to complete the proof. Hence, we may freely assume $Z$ is not a subset of $S^{+}$and is instead a subset of $S^{-}$. Similarly, define $T^{+}$and $T^{-}$such that $X \subset T^{+}$and $Z \subset T^{-}$.

As $S \neq T$, there is $y \in T \backslash S$. Further, there is an open neighborhood $U$ containing $y$ which lies entirely in the interior of either $S^{-}$or $S^{+}$and intersects the interiors of both $T^{+}$and $T^{-}$. This means that at least one of $S^{+} \cap T^{-}$and $S^{-} \cap T^{+}$has non-empty interior. As both of these intersections are unions of leaves of $\mathcal{F}^{c s}$ which lie entirely in $Y$, this shows that $\Lambda$ has non-empty interior. 
For an unstable arc $J$, define $U_{1}^{c s}(J)$ as all points $x \in \tilde{M}_{A}$ such that $\operatorname{dist}(x, J)<1$ and every leaf of $\mathcal{F}^{c s}$ which contains $x$ also intersects $J$.

Lemma 5.5. There is $C>0$ such that

$$
\text { volume }\left(\operatorname{int}\left(U_{1}^{c s}(J)\right)\right) \geq C \text { length }(J)
$$

for all unstable arcs $J$ of length at least one.

This is a slight modification of Lemma 3.3 of $\left[\mathrm{BBI}_{2}\right]$. For completeness, we give a short proof.

Proof. As the distributions $E^{c s}$ and $E^{u}$ are uniformly continuous and uniformly transverse, there is $\delta>0$ such that the volume of the interior of $U_{1}^{c s}(J)$ is greater than $\delta$ for any unstable curve $J$ of length at least one. If length $(J)>n$, there are $n$ disjoint subcurves $J_{1}, \ldots, J_{n} \subset J$ of length one. If $U_{1}^{c s}\left(J_{i}\right)$ intersected $U_{1}^{c s}\left(J_{j}\right)$ at a point $x$, then any leaf of $\mathcal{F}^{c s}$ through $x$ would intersect $J$ at distinct points in $J_{i}$ and $J_{j}$ contradicting Proposition 3.3 .

Proof of Lemma 5.3. If the claim is false, then by Lemma 5.4, there is a leaf $\mathcal{L} \in \mathcal{A}^{\text {cs }}$ such that the corresponding union of leaves $\Lambda$ has non-empty interior.

If $\gamma \in G_{0}$ and the interiors of $\Lambda$ and $\gamma(\Lambda)$ intersected, there would be a leaf $L \in \mathcal{F}^{c s}$ such that both $d_{H}(L, \mathcal{L})<\infty$ and $d_{H}(L, \gamma(\mathcal{L}))<\infty$ and therefore $\mathcal{L}=\gamma(\mathcal{L})$ by Lemma 5.2. One can check from the definitions of $G_{0}$ and $\mathcal{A}^{c s}$ in the previous section that there is no such leaf with this property. Hence, by Lemma 4.8, the volume of

$$
X_{n}:=\left\{x \in \operatorname{int}(\Lambda):\left|p_{1}(x)\right|<n\right\}
$$

grows linearly in $n$.

By Lemma 5.2, we have $f(\Lambda)=\Lambda$. Take an unstable arc $J$ in the interior of $\Lambda$. As $f$ is a finite distance from the identity, there is $N$ such that for all $k \geq 1, f^{k}(J)$ is contained in a ball of radius $N k$ and therefore $U_{1}^{c s}\left(f^{k}(J)\right)$ is contained in a ball of radius $N k+1$. By Lemma 4.2, there is $C>0$ such that $\left|p_{1}(x)\right|<N k+C$ for all $x \in U_{1}^{c s}\left(f^{k}(J)\right)$.

In other words, $\operatorname{int}\left(U_{1}^{c s}\left(f^{k}(J)\right)\right) \subset X_{N k+C}$ for all $k \geq 1$. Since the length of $f^{k}(J)$ grows exponentially fast in $k$, and the volume of $X_{N k+C}$ grows only linearly, Lemma 5.5 gives a contradiction.

This leads to the following consequence which is of critical importance to the overall proof.

Corollary 5.6. If $L \in \mathcal{F}^{c s}$, then $f(L)=L$.

Proof. This follows from Lemmas 5.3 and 5.2 . 


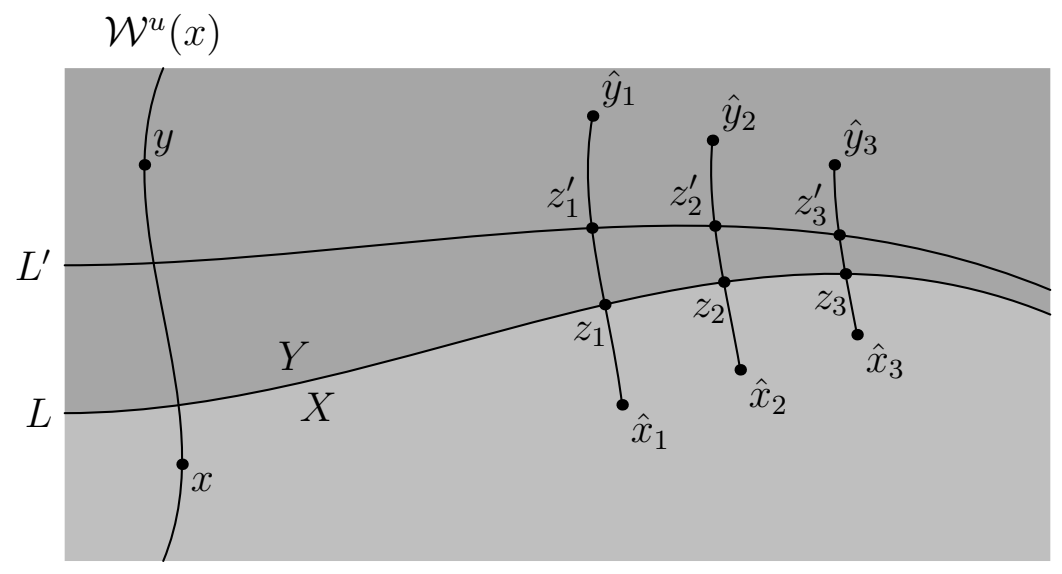

FiguRE 3. A depiction of the sequences in the proof of Lemma 5.8 .

Now knowing that leaves of $\mathcal{F}^{c s}$ are fixed by $f$, we now proceed to prove that $\mathcal{F}^{c u}$ is almost parallel to $\mathcal{A}^{c u}$.

Lemma 5.7. If $\left\{z_{k}\right\},\left\{z_{k}^{\prime}\right\}$ are sequences in leaves $L, L^{\prime} \in \mathcal{F}^{c s}$ such that $\sup d\left(z_{k}, z_{k}^{\prime}\right)<\infty$ and $\lim _{k \rightarrow \infty} p_{1}\left(z_{k}\right)=+\infty$, then $L=L^{\prime}$.

Proof. Let $\mathcal{L}, \mathcal{L}^{\prime} \in \mathcal{A}^{c s}$ be such that $d_{H}(L, \mathcal{L})<R$ and $d_{H}\left(L^{\prime}, \mathcal{L}^{\prime}\right)<R$. Then, there are sequences $\left\{x_{k}\right\}$ and $\left\{x_{k}^{\prime}\right\}$ in $\mathcal{L}$ and $\mathcal{L}^{\prime}$ such that $d\left(x_{k}, z_{k}\right)<R$ and $d\left(x_{k}^{\prime}, z_{k}^{\prime}\right)<R$ for all k. By Lemma 4.4 and the triangle inequality, $\mathcal{L}=\mathcal{L}^{\prime}$. By Lemma 5.3, $L=L^{\prime}$.

Lemma 5.8. There is $K>0$ such that

$$
p_{1}\left(f^{-n}(x)\right)-p_{1}(x)<K
$$

for all $x \in \tilde{M}_{A}$ and $n \geq 0$.

Proof. Suppose the statement is false. Then there are sequences $\left\{x_{k}\right\},\left\{\hat{x}_{k}\right\}$ in $\tilde{M}_{A}$ and $\left\{n_{k}\right\}$ in $\mathbb{N}$ such that $\hat{x}_{k}=f^{-n_{k}}\left(x_{k}\right)$ for all $k$ and $\lim _{k} p_{1}\left(\hat{x}_{k}\right)-p_{1}\left(x_{k}\right)=+\infty$. By Lemma 4.1, one may freely assume that $x_{k}$ lies within a compact fundamental domain. By taking a subsequence, further assume that $x_{k}$ converges to a point $x$. Then, $\lim _{k} p_{1}\left(\hat{x}_{k}\right)=+\infty$.

Define a convergent sequence $\left\{y_{k}\right\}$ such that each $y_{k}$ is connected to $x_{k}$ by an unstable segment of length exactly one. Then $y:=\lim _{k} y_{k}$ is on the unstable leaf of $x$ and is distinct from $x$. Define $\hat{y}_{k}:=f^{-n_{k}}\left(y_{k}\right)$. As $f^{-1}$ contracts unstable leaves, $\sup d\left(\hat{x}_{k}, \hat{y}_{k}\right)<$ $\infty$.

Choose a leaf $L \in \mathcal{F}^{c s}$ which intersects $\mathcal{W}^{u}(x)$ at a point strictly between $x$ and $y$. This leaf splits $\tilde{M}_{A}$ into closed half-spaces $X$ and $Y$ such that $\partial X=\partial Y=X \cap Y=L$ and $x \in \operatorname{int}(X)$ and $y \in \operatorname{int}(Y)$. 
For large $k, x_{k} \in \operatorname{int}(X)$. By Lemma 5.6, $\hat{x}_{k}$ is on the same leaf as $x_{k}$ and as leaves do not cross topologically, $\hat{x}_{k} \in X$. Similarly, $\hat{y}_{k} \in Y$ for large $k$. By taking subsequences, assume "large $k$ " is all $k \geq 1$.

As any path connecting $\hat{x}_{k}$ to $\hat{y}_{k}$ must intersect $L$, there is a sequence $\left\{z_{k}\right\}$ in $L$ such that

$$
\sup d\left(\hat{x}_{k}, z_{k}\right) \leq \sup d\left(\hat{x}_{k}, \hat{y}_{k}\right)<\infty
$$

which also implies $\lim p_{1}\left(z_{k}\right)=+\infty$. Now choose a leaf $L^{\prime} \in \mathcal{F}^{c s}$ which intersects the unstable segment between $x$ and $y$ at a point distinct from $L$. By the same construction, there is a sequence $\left\{z_{k}^{\prime}\right\}$ in $L^{\prime}$ such that $\sup d\left(\hat{x}_{k}, z_{k}^{\prime}\right)<\infty$ and therefore $\sup d\left(z_{k}, z_{k}^{\prime}\right)<\infty$. By Lemma 5.7, $L$ and $L^{\prime}$ are the same leaf, which by Proposition 3.3 gives a contradiction.

We now have the tools necessary to compare $\mathcal{F}^{c u}$ and $\mathcal{A}^{c u}$.

Proposition 5.9. The branching foliation $\mathcal{F}^{c u}$ is almost parallel to $\mathcal{A}^{c u}$.

Proof. $\mathcal{F}^{c u}$ is almost parallel either to $\mathcal{A}^{c u}$ or $\mathcal{A}^{c s}$. Assume the latter in order to derive a contradiction. Notice that a center-unstable branching foliation for a partially hyperbolic diffeomorphism is a center-stable branching foliation for its inverse. That is, if $g=f^{-1}$, then $\mathcal{F}_{f}^{c u}=\mathcal{F}_{g}^{c s}$ and so the assumption is that $\mathcal{F}_{g}^{c s}$ is almost parallel to $\mathcal{A}^{c s}$. Repeating all of the results of this section with $g$ in place of $f$, one proves a version of Lemma 5.8 for $g$ :

There is $K>0$ such that

$$
p_{1}\left(f^{n}(x)\right)-p_{1}(x)=p_{1}\left(g^{-n}(x)\right)-p_{1}(x)<K
$$

for all $x \in \tilde{M}_{A}$ and $n \geq 0$.

Taken with the original Lemma 5.8 for $f$, this implies that there is $K$ such that

$$
\left|p_{1}\left(f^{n}(x)\right)-p_{1}(x)\right|<K
$$

for all $x \in \tilde{M}_{A}$ and $n \in \mathbb{Z}$.

Take an unstable curve $J$ such that $J \subset \tilde{V}_{1}$ in the notation of Lemma 4.7. The above inequality implies that $f^{n}(J) \subset \tilde{V}_{K+1}$ for all $n$ and therefore $U_{1}^{c s}\left(f^{n}(J)\right) \subset \tilde{V}_{K+2}$ (where $U_{1}^{c s}$ is defined just before Lemma 5.5). Arguing as in the proof of Lemma 5.3, the volume of $U_{1}^{c s}\left(f^{n}(J)\right)$ grows exponentially, but its diameter grows only linearly. This contradicts the polynomial growth of volume given in Lemma 4.7.

\section{Dynamical COHEREnCE}

This section gives the proof of dynamical coherence. The assumptions are exactly the same as in the previous section. The starting point of the proof is the following consequence of Proposition 5.9: 
Lemma 6.1. There is $N>0$ such that for every $L^{c s} \in \mathcal{F}^{c s}$ and $L^{c u} \in \mathcal{F}^{c u}$, the intersection $L^{c s} \cap L^{c u}$ consists of at most $N$ connected components, each of which is a properly embedded line.

Proof. Each component of $L^{c s} \cap L^{c u}$ is a one-dimensional manifold in $L^{c s}$ transverse to the unstable foliation $\mathcal{W}^{s}$ restricted to $L^{c s}$. Since no circle can be transverse to a foliation of a plane by lines, each component is a line. If a component is not properly embedded, it accumulates at a point $y \in L^{c u}$ and $\mathcal{W}^{u}(y)$ intersects $L^{c s}$ more than once, contradicting Proposition 3.3. Distinct components $C_{1}$ and $C_{2}$ must be bounded away from each other by some uniform distance $\epsilon>0$, as otherwise a single unstable leaf would intersect both $C_{1}$ and $C_{2}$ and again give a contradiction.

By Lemma 5.2 (and its cu counterpart) and Proposition 5.9, there are unique leaves $\mathcal{L}^{c s} \in \mathcal{A}^{c s}$ and $\mathcal{L}^{c u} \in \mathcal{A}^{c u}$ such that $d_{H}\left(L^{c s}, \mathcal{L}^{c s}\right)<R$ and $d_{H}\left(L^{c u}, \mathcal{L}^{c u}\right)<R$. For any $t \in \mathbb{R}$, define the disk

$$
D_{t}:=\left\{x \in \tilde{M}_{A}: \operatorname{dist}\left(x, \mathcal{L}^{c s}\right)<R, \operatorname{dist}\left(x, \mathcal{L}^{c u}\right)<R, p_{1}(x)=t\right\} .
$$

By Lemma 4.3 (and its cu counterpart), the diameter of $D_{t}$ is finite and depends purely on $R$ and not $\mathcal{L}^{c u}, \mathcal{L}^{c s}$, or $t$. In particular, there is $K>0$ such that any subset of $D_{t}$ with at least $K$ points has two points at distance less than $\epsilon$.

Since a connected component $C$ of $L^{c s} \cap L^{c u}$ is properly embedded, its image $p_{1}(C) \subset \mathbb{R}$ is of the form $[a,+\infty),(-\infty, a]$, or $\mathbb{R}$. If there are more than $2 K$ components, then there is $t \in \mathbb{R}$ such that $t \in p_{1}\left(C_{i}\right)$ for $K$ different components $C_{1}, \ldots, C_{K}$. Each $C_{i}$ then intersects $D_{t}$, and this gives a contradiction.

The following key property in the proof is inspired by a similar argument due to Bonatti and Wilkinson (see Corollary 3.11 of $[\mathrm{BoW}]$ ):

Lemma 6.2. The diffeomorphism $f$ has no periodic points.

Remark. Here, as in all of this section, this is a statement about the map $f$ on the universal cover $\tilde{M}_{A}$. A partially hyperbolic map on the compact manifold $M_{A}$ can certainly have periodic points.

Proof. Suppose $f^{n}(x)=x$. Take $y \in \mathcal{W}^{u}(x)$ and $L \in \mathcal{F}^{c s}$ such that $y \in L$. As $f(L)=L$ and $y$ is the unique intersection of $\mathcal{W}^{u}(x)$ and $L$, it follows that $f^{n}(y)=y$. This contradicts the fact that $f^{-1}$ must contract the unstable segment between $x$ and $y$.

Lemma 6.3. For all $x \in \tilde{M}_{A}, \lim _{n \rightarrow \infty} p_{1}\left(f^{n}(x)\right)=+\infty$.

Proof. The point $x$ lies on a connected component $C$ of a set of the form $L^{c s} \cap L^{c u}$. By Lemma 6.1, there is $n>0$ such that $f^{n}(C)=C$ and by Lemma 6.2 this map has no fixed points. By the properties of fixed-point free homeomorphisms of the real 
line, the orbit $f^{n k}(x)$ must eventually leave any compact subset of $C$. Since for any $T>0$, one can show that the set $L^{c s} \cap L^{c u} \cap p_{1}^{-1}([-T, T])$ is compact, this implies that $\lim _{k \rightarrow \infty}\left|p_{1}\left(f^{n k}(x)\right)\right|=\infty$ and from Lemma 5.8 the result follows.

Proposition 6.4. The branching foliation $\mathcal{F}^{c s}$ is the unique f-invariant, G-invariant branching foliation tangent to $E^{c s}$ and it is a true foliation.

Proof. Define a map $H: \tilde{M}_{A} \rightarrow \mathcal{A}^{c s}$ by requiring that

$$
\sup _{n \geq 0} \operatorname{dist}\left(f^{n}(x), H(x)\right)<\infty \text {. }
$$

Using Corollary 5.6 and Lemmas 5.2, 6.3, and 4.4, one can check that $H$ is well-defined and its fibers $H^{-1}(\{\mathcal{L}\})$ are exactly the leaves of $\mathcal{F}^{c s}$. Since the definition of $H$ does not use $\mathcal{F}^{c s}$, this shows there is only one such possible branching foliation. As the fibers are disjoint, Proposition 3.4 shows that $\mathcal{F}^{c s}$ is a true foliation.

Taken with the corresponding statement for the $c u$ foliation, Proposition 6.4 implies dynamical coherence and completes the proof of Lemma 2.1. Modulo the proof of Proposition 1.3, which is left to Appendix A, this also establishes Proposition 1.4 and Theorem A.

\section{LEAF CONJUGACY}

This section gives the proof of Proposition 1.5. Assume $f_{0}: M_{A} \rightarrow M_{A}$ satisfies the hypotheses of Proposition [1.5. As $f_{0}$ is homotopic to the identity, it lifts to a function $f: \tilde{M}_{A} \rightarrow \tilde{M}_{A}$ which is a finite distance from the identity. As $f_{0}$ is dynamically coherent, there are $f_{0}$-invariant (non-branching) foliations tangent to $E^{c s}$ and $E^{c u}$ and, by a result announced in $\left[\mathrm{RHRHU}_{2}\right.$, these foliations do not have compact leaves. Therefore, Proposition 3.7 applies and these foliations lift to foliations on $\tilde{M}_{A}$ which are almost parallel either to $\mathcal{A}^{c s}$ or $\mathcal{A}^{c u}$.

The proofs in Sections 5 and 6 now follow as before, with these true foliations taking the place of the branching foliations. There is, however, one small caveat. It is possible for $f_{0}$ to be both dynamically coherent and to have an invariant torus tangent to $E^{c s}$ or $E^{c u}$. (See $\mathrm{RHRHU}_{2}$ for an example.) As such, there may be a branching foliation on $\tilde{M}_{A}$ with a leaf which quotients down to a compact surface on $M_{A}$. Such a branching foliation will not be almost parallel to $\mathcal{A}^{c s}$ or to $\mathcal{A}^{c u}$. This issue only affects the proof of uniqueness in Proposition 6.4. Since Proposition 6.4 is not needed to establish the leaf conjugacy, this is not an issue in proving Proposition 1.5.

Proposition 7.1. There is $n_{0}>0$ such that $p_{1}\left(f^{n}(x)\right)>p_{1}(x)+1$ for all $x \in \tilde{M}_{A}$ and $n>n_{0}$. 
Proof. By Lemma 4.1, it is enough to prove this for a compact fundamental domain. By Lemma 6.3, the sets

$$
U_{n}=\left\{x \in \tilde{M}_{A}: p_{1}\left(f^{n}(x)\right)-p_{1}(x)>K+1\right\} .
$$

for $n \in \mathbb{N}$ form an open cover of $\tilde{M}_{A}$. Thus, some finite union $U_{n_{1}} \cup \cdots \cup U_{n_{k}}$ covers a fundamental domain. One can then show using Lemma 5.8 that $n_{0}=\max \left\{n_{1}, \ldots, n_{k}\right\}$ satisfies the desired property.

By adapting a proof of Fuller regarding flows $[\mathrm{Fu}$, we prove the following.

Lemma 7.2. There is a continuous function $p_{c}: \tilde{M}_{A} \rightarrow \mathbb{R}$ such that

- for each leaf $L \in \mathcal{F}^{c}$, the restriction $\left.p_{c}\right|_{L}: L \rightarrow \mathbb{R}$ is a $C^{1}$ diffeomorphism,

- for each $t \in \mathbb{R}$, the level set $p_{c}^{-1}(\{t\})$ is an embedded $C^{0}$ surface in $\tilde{M}_{A}$, and

- $p_{c}(\gamma(x))-p_{c}(x)=p_{1}(\gamma(x))-p_{1}(x)$ for all $\gamma \in G$ and $x \in \tilde{M}_{A}$.

Proof. By Lemma 6.1 and Proposition 7.1, there is $n>0$ such for all $x \in \tilde{M}_{A}, f^{n}(x)$ is on the same center leaf as $x$ and $p_{1}\left(f^{n}(x)\right)>p_{1}(x)+1$. By a compactness argument, the distance $d_{c}\left(x, f^{n}(x)\right)$ along the center leaf is uniformly bounded both above and away from zero. Consequently, there is $T>0$ such that if $x$ and $y$ are on the same center leaf and $d_{c}(x, y)>T$, then $\left|p_{1}(x)-p_{1}(y)\right|>1$.

For a leaf $L \in \mathcal{F}^{c}$, let $\alpha: \mathbb{R} \rightarrow L$ be a path parameterizing $L$ by arc length and oriented such that $p_{1}(\alpha(t)) \rightarrow+\infty$ as $t \rightarrow+\infty$. Define $p_{c}$ on $L$ by

$$
p_{c}(\alpha(t))=\frac{1}{T} \int_{t}^{t+T} p_{1}(\alpha(s)) d s .
$$

By the Fundamental Theorem of Calculus,

$$
\frac{d}{d t} p_{c}(\alpha(t))=\frac{1}{T}\left[p_{1}(\alpha(t+T))-p_{1}(\alpha(t))\right]>\frac{1}{T}
$$

so that $p_{c} \circ \alpha$ is a $C^{1}$ diffeomorphism. From this, the above listed properties are easily verified.

Recall from Section 4 that points in $\tilde{M}_{A}$ can be written in coordinates $(v, t)$ with $v \in \mathbb{R}^{2}$ and $t \in \mathbb{R}$ and that the group $G$ of deck transformations is generated by the isometries $\gamma_{i}: \tilde{M}_{A} \rightarrow \tilde{M}_{A}(i=1,2,3)$. In these coordinates, $\gamma_{3}^{-1}(v, t)=(A v, t-1)$. Define a surface $\tilde{S}=p_{c}^{-1}(\{0\}) \subset \tilde{M}_{A}$ and a map $\psi: \tilde{S} \rightarrow \tilde{S}$ by $\psi(x)=\gamma_{3}^{-1}(y)$ where $y$ is the unique point in $\mathcal{F}^{c}(x)$ such that $p_{c}(y)=1$.

One can show that if $(v, t) \in \tilde{S}$, then $(v+z, t) \in \tilde{S}$ for $z \in \mathbb{Z}^{2}$ and

$$
\psi(v+z, t)=\psi(v, t)+(A v, 0) .
$$

Now quotient $\psi$ down to a map $\psi_{0}: S \rightarrow S$ on $S \subset M_{A}$. The surface $S$ is homeomorphic to a 2 -torus and the induced action of of $\psi_{0}$ on the fundamental group of $S$ is given by the linear map $A$. 
Lemma 7.3. The map $\psi$ is expansive.

Proof. Suppose that for $\epsilon>0$ there are points $x$ and $y$ such that $d\left(\psi^{n}(x), \psi^{n}(y)\right)<\epsilon$ for all $n \in \mathbb{Z}$. Then, $d\left(\gamma_{3}^{n} \psi^{n}(x), \gamma_{3}^{n} \psi^{n}(y)\right)<\epsilon$ and Lemma 5.7 shows that $x$ and $y$ lie on the same leaf $L^{c s}$ of $\mathcal{F}^{c s}$. An analogous argument shows that they lie on the same leaf of $\mathcal{F}^{c u}$. If $x$ and $y$ lie on distinct center leaves $L_{x}, L_{y} \in \mathcal{F}^{c}$ and if $\epsilon$ is sufficiently small, then there is an unstable arc connecting $L_{x}$ to $L_{y}$. This arc intersects $L^{c s}$ in distinct points, contradicting Lemma 3.3. Hence, $x$ and $y$ lie on the same center leaf, and since $\tilde{S}$ intersects each center leaf exactly once, $x=y$.

Remark. Using Proposition 7.1 and Lemma 7.3, it is easy to show that $f$ is plaque expansive as defined in [HPS].

Proposition 7.4. The quotient $f_{0}: M_{A} \rightarrow M_{A}$ of $f: \tilde{M}_{A} \rightarrow \tilde{M}_{A}$ is leaf conjugate to the time-one map of a suspension Anosov flow.

Proof. By a result of Lewowicz, any expansive homeomorphism of a torus is topologically conjugate to a linear Anosov diffeomorphism $[\mathrm{L}$. The linear diffeomorphism is determined by the action on the fundamental group.

In the case of $\psi$ and its quotient $\psi_{0}$, this implies that there is a homeomorphism $H: \tilde{S} \rightarrow \mathbb{R}^{2}$ so that $H \circ \psi=A \circ H$ and $H(v+z, t)=H(v, t)+(A z, 0)$ for all $z \in \mathbb{Z}^{2}$.

Now define a unique homeomorphism $h: \tilde{M}_{A} \rightarrow \tilde{M}_{A}$ by requiring

(1) that the leaf of $\mathcal{F}^{c}$ through the point $x \in \tilde{S}$ is mapped to the leaf of $\mathcal{A}^{c}$ through the point $(H(x), 0) \in \tilde{M}_{A}$, and

(2) that $p_{c} \circ h=p_{1}$.

By its construction, $h$ maps $\mathcal{F}^{c}$ to $\mathcal{A}^{c}$. Suppose $x, y \in \tilde{S}$ are such that $H(x)$ and $H(y)$ are on the same stable leaf of $A$. By the conjugacy, $d\left(\psi^{n}(x), \psi^{n}(y)\right) \rightarrow 0$ as $n \rightarrow \infty$, which implies that $d\left(\gamma_{3}^{n} \psi^{n}(x), \gamma_{3}^{n} \psi^{n}(y)\right) \rightarrow 0$. As $\psi$ was defined in such a way that $\gamma_{3} \psi(x)$ is on the same center leaf as $x$, it follows from Lemma 5.7 that $x$ and $y$ are on the same leaf of $\mathcal{F}^{c s}$. Hence, $h\left(\mathcal{F}^{c s}\right)=\mathcal{A}^{c s}$. Similarly, $h\left(\mathcal{F}^{c u}\right)=\mathcal{A}^{c u}$.

Since each leaf of $\mathcal{A}^{c s}$ intersects each leaf of $\mathcal{A}^{c u}$ in a single leaf of $\mathcal{A}^{c}$, applying $h^{-1}$ shows that a leaf of $\mathcal{F}^{c s}$ intersects a leaf of $\mathcal{F}^{c u}$ in a single leaf of $\mathcal{F}^{c}$. Consequently, $f$ fixes all leaves of $\mathcal{F}^{c}$, and $h$ is a leaf conjugacy between $f$ and the time-one map $(v, t) \mapsto(v, t+1)$ of the lifted Anosov flow on $\tilde{M}_{A}$.

This quotients down to a leaf conjugacy on the compact manifold $M_{A}$.

This finishes the proof of leaf conjugacy for partially hyperbolic diffeomorphisms which are homotopic to the identity (Proposition 1.5). Up to Proposition 1.3 which is proved in Appendix $\mathrm{A}$ this completes the proof of the Main Theorem. 
Further, the homeomorphism $h$ constructed in the last proof has several useful properties listed here.

Lemma 7.5. There is a homeomorphism $h: \tilde{M}_{A} \rightarrow \tilde{M}_{A}$ such that

- $h\left(\mathcal{F}^{\sigma}\right)=\mathcal{A}^{\sigma}$ for $\sigma=c, c s, c u$,

- $h \circ \gamma=\gamma \circ h$ for all $\gamma \in G$,

- $\sup _{x \in \tilde{M}_{A}} d(x, h(x))<\infty$,

- $h$ and $h^{-1}$ are uniformly continuous.

To end the section, we use these properties to prove Global Product Structure, a property of the foliations which does not immediately follow from the leaf conjugacy.

Proposition 7.6. $f$ has Global Product Structure. That is, for $x, y \in \tilde{M}_{A}$

(1) $\mathcal{F}^{c s}(x)$ intersects $\mathcal{W}^{u}(y)$ in a unique point,

(2) $\mathcal{F}^{c u}(x)$ intersects $\mathcal{W}^{s}(y)$ in a unique point,

(3) if $x \in \mathcal{F}^{c s}(y)$, then $\mathcal{F}^{c}(x)$ intersects $\mathcal{W}^{s}(y)$ in a unique point,

(4) if $x \in \mathcal{F}^{c u}(y)$, then $\mathcal{F}^{c}(x)$ intersects $\mathcal{W}^{u}(y)$ in a unique point.

Proof. The uniqueness of the intersections follows from Proposition 3.3 (and its analogous statement for $\mathcal{F}^{c u}$ and $\mathcal{W}^{s}$ ). Therefore, it is enough to prove existence. As each leaf of $\mathcal{F}^{c s}$ intersects each leaf of $\mathcal{F}^{c u}$ in a single leaf of $\mathcal{F}^{c}$, items (1) and (2) will follow from (3) and (4).

To prove existence of the intersection in (3), suppose $L \in \mathcal{F}^{c}, L \subset L^{c s} \in \mathcal{F}^{c s}$ and $x \in L^{c s}$. Then $h\left(f^{n}(x)\right)$ is on the leaf $\mathcal{A}^{c}(h(x))$ for all $n$, and by Lemmas 4.2, 6.3, and 7.5

$$
\lim _{n \rightarrow \infty} p_{1}\left(h\left(f^{n}(x)\right)\right)=+\infty .
$$

By the definition of $\mathcal{A}^{c}$ and $\mathcal{A}^{c s}, \lim _{n \rightarrow \infty} \operatorname{dist}\left(h\left(f^{n}(x)\right), \mathcal{L}\right)=0$ for any leaf $\mathcal{L} \in \mathcal{A}^{c}$ where $\mathcal{L} \subset \mathcal{A}^{c s}(h(x))$. In particular, if $\mathcal{L}=h(L)$, then by the uniform continuity of $h^{-1}$, $\lim _{n \rightarrow \infty} \operatorname{dist}\left(f^{n}(x), L\right)=0$. Consequently, there is $n$ such that $f^{n}(x)$ is close enough to $L$ that $\mathcal{W}^{s}\left(f^{n}(x)\right)$ intersects $L$. Applying $f^{-n}$ shows that $\mathcal{W}^{s}(x)$ intersects $L$ and $(3)$ is proved. The proof of (4) is analogous.

\section{Appendix A. Quotients of PARTIALly hyperbolic DifFEOMORPhisms}

This appendix studies the algebraic properties of partially hyperbolic systems on solvmanifolds and is divided into three subsections. The first classifies partially hyperbolic systems on suspension manifolds when they are not homotopic to the identity and gives the proof of Proposition 1.3. The second subsection proves Proposition 1.2. The last subsection classifies partial hyperbolicity on quotients of the 3-torus and other nilmanifolds. 
A.1. Algebra and leaf conjugacies. Since $\tilde{M}_{A}$ is homeomorphic to $\mathbb{R}^{3}$, if a diffeomorphism on $M_{A}$ is not homotopic to the identity, then it will induce a non-trivial isomorphism of $G=\pi_{1}\left(M_{A}\right)$. We first study these isomorphisms and their corresponding algebraic maps on $\tilde{M}_{A}$.

Recall a point of $\tilde{M}_{A}$ can be written as $(x, t)$ with $x \in \mathbb{R}^{2}$ and $t \in \mathbb{R}$. For $z \in \mathbb{Z}^{2}$, let $\alpha_{z}$ denote the map $\alpha_{z}(x, t)=(x+z, t)$. Then, $G_{0}=\left\{\alpha_{z}: z \in \mathbb{Z}^{2}\right\}$ is a normal subgroup of $G$, and $G$ is generated by elements of $G_{0}$ together with the deck transformation $\gamma_{3}(x, t)=\left(A^{-1} x, t+1\right)$.

Lemma A.1. For any isomorphism $\phi: G \rightarrow G$ there are $B \in G L(2, \mathbb{Z}), v \in \mathbb{Z}^{2}$, and $e \in\{+1,-1\}$ such that $\phi\left(\alpha_{z}\right)=\alpha_{B z}$ for $z \in \mathbb{Z}$ and $\phi\left(\gamma_{3}\right)=\alpha_{v} \gamma_{3}^{e}$. Moreover, $A^{e} B=B A$ and $B^{j}= \pm A^{k}$ for some $j, k \in \mathbb{Z}$ with $j>0$.

See the appendix of [GS] for a proof of the main statement of the lemma. The fact that $B^{j}= \pm A^{k}$ is a consequence of the following classical result.

Lemma A.2. If $A \in G L(2, \mathbb{Z})$ is hyperbolic, then there is $A_{0} \in G L(2, \mathbb{Z})$ such that $B A=A B$ if and only if $B$ is of the form $\pm A_{0}^{k}$ for some $k \in \mathbb{Z}$.

See, for instance, $[\mathrm{BR}]$ for a proof.

Due to Lemma A.1, every automorphism of $G$ is induced by an algebraic map on $\tilde{M}_{A}$.

Lemma A.3. For any automorphism $\phi: G \rightarrow G$ there are unique $B \in G L(2, \mathbb{Z})$, $w \in \mathbb{R}^{2}$, and $e \in\{+1,-1\}$ such that the diffeomorphism

$$
\Phi: \tilde{M}_{A} \rightarrow \tilde{M}_{A}, \quad(x, t) \mapsto(B x+w, e t)
$$

satisfies $\Phi \circ \gamma=\phi(\gamma) \circ \Phi$ for all $\gamma \in G$. As such, $\Phi$ quotients to a map $\Phi_{0}: M_{A} \rightarrow M_{A}$. If $e=+1$, then $\Phi\left(\mathcal{A}^{c s}\right)=\mathcal{A}^{c s}$ and $\Phi\left(\mathcal{A}^{c u}\right)=\mathcal{A}^{c u}$. If $e=-1$, then $\Phi\left(\mathcal{A}^{c s}\right)=\mathcal{A}^{c u}$ and $\Phi\left(\mathcal{A}^{c u}\right)=\mathcal{A}^{c s}$.

Proof. Let $B, v, e$ be as in Lemma A.1. Let $w \in \mathbb{R}^{2}$ solve the equation $A^{-e} w+v=w$. Such a solution exists and is unique as $A$ is hyperbolic. Then, one can verify directly that $\Phi \circ \gamma=\phi(\gamma) \circ \Phi$. If $A B=B A$, then $B$ preserves the eigenspaces of $A$. If $A^{-1} B=B A$, then $B$ maps an eigenspace $E_{\lambda}$ of $A$ to the eigenspace $E_{\lambda^{-1}}$. From this, the claims about $\Phi\left(\mathcal{A}^{c s}\right)$ and $\Phi\left(\mathcal{A}^{c u}\right)$ follow.

Lemma A.4. For any such $\Phi$, there is an iterate $n>0$ which is of the form

$$
\Phi^{n}(x, t)=\left(A^{m} x+z, t\right)
$$

where $m \in \mathbb{Z}$ and $z \in \mathbb{Z}^{2}$.

Proof. The equation $A^{-e} w+v=w$ from the last proof can be written as a system of linear equations with integer coefficients and can therefore be solved over $\mathbb{Q}$. This means 
that $x \mapsto B x+w$ defines a permutation of a finite set of the form $\mathbb{Z} / q \times \mathbb{Z} / q$ for some $q$. As some iterate $\ell$ of this permutation fixes $(0,0)$ the corresponding function on $\mathbb{R}^{2}$ is of the form $x \mapsto B^{\ell} x+z$ for some $z \in \mathbb{Z}^{2}$. Suppose $B^{j}= \pm A^{k}$. Then, $\Phi^{n}$ for $n=2 j \ell$ will have the desired form.

Corollary A.5. If $\Phi_{0}: M_{A} \rightarrow M_{A}$ is an algebraic map as in Lemma A.3, then there is $n \geq 1$ such that $\Phi_{0}^{n}$ is homotopic to the identity.

Proof. One may write $M_{A}$ as the quotient $\mathbb{T}^{2} \times \mathbb{R} / \sim$ where $(A x, t) \sim(x, t+1)$. Then by Lemma A.4, $\Phi_{0}^{n}: M_{A} \rightarrow M_{A}$ can be written as $\Phi_{0}^{n}(x, t)=\left(A^{m} x+z, t\right)=(x, t+m)$ which is clearly homotopic to the identity.

From this, we can now prove Proposition 1.3.

Proof of Proposition 1.3. If $f: \tilde{M}_{A} \rightarrow \tilde{M}_{A}$ descends to $f_{0}: M_{A} \rightarrow M_{A}$, then by Lemma A.3, there is $\Phi$ descending to $\Phi_{0}$ such that $f$ and $\Phi$ induce the same automorphism of $\pi_{1}\left(M_{A}\right)$. As $M_{A}$ is $K(\pi, 1)$, Whitehead's theorem implies that $f_{0}$ and $\Phi_{0}$ are homotopic. By Corollary A.5, there is $n$ such that $f_{0}^{n}$ is homotopic to the identity.

If the $B$ in Lemma A.3 is hyperbolic, the corresponding $\Phi$ is partially hyperbolic. If $B= \pm I d$, one can compose $\Phi$ with a flow along the center foliation $\mathcal{A}^{c}$ to produce a partially hyperbolic map. In any case, we say a diffeomorphism $g: \tilde{M}_{A} \rightarrow \tilde{M}_{A}$ which preserves foliations $\mathcal{F}^{c u}, \mathcal{F}^{c s}, \mathcal{F}^{c}$ is leaf conjugate to $\Phi$ if there is a homeomorphism $h: \tilde{M}_{A} \rightarrow \tilde{M}_{A}$ such that $h\left(\mathcal{F}^{\sigma}\right)=\mathcal{A}^{\sigma}$ and

$$
h(f(L))=\Phi(h(L))
$$

for all $L \in \mathcal{F}^{\sigma}$ and $\sigma=c, c s, c u$. If $g, \Phi, h$ quotient to maps $g_{0}, \Phi_{0}, h_{0}$ on $M_{A}$, we say $g_{0}$ and $\Phi_{0}$ are leaf conjugate as well.

Theorem A.6. Suppose $g_{0}: M_{A} \rightarrow M_{A}$ is partially hyperbolic and there is no periodic torus tangent to $E^{c s}$ or $E^{c u}$. Then $g_{0}$ is dynamically coherent and is leaf conjugate to an algebraic map $\Phi_{0}$ as given in Lemma A.3 with $e=+1$.

Proof. Dynamical coherence follows from Proposition 1.4, so we need only establish the leaf conjugacy. Choose a lift $g: \tilde{M}_{A} \rightarrow \tilde{M}_{A}$ of $g_{0}$. This defines an isomorphism $g_{*}=\phi: G \rightarrow G$ by

$$
g \circ \gamma=\phi(\gamma) \circ g
$$

Let $\Phi$ be the corresponding algebraic map given by Lemma A.3. Note that the function $x \mapsto d(g(x), \Phi(x))$ quotients down to $M_{A}$ and is therefore bounded. This shows

$$
\sup _{x \in \tilde{M}_{A}} d(g(x), \Phi(x))<\infty
$$


By Lemma A.4, there is $n$ such that $\Phi^{n}(v, t)=\left(A^{k} v+z, t\right)$ for some $k$ and $z$. Define $\hat{\gamma}=\gamma_{3}^{k} \circ \alpha_{z}, f=\hat{\gamma} \circ g^{n}$, and $\Phi_{f}=\hat{\gamma} \circ \Phi^{n}$. One can check from the definitions of $\alpha_{z}$ and $\gamma_{3}$ that $\Phi_{f}(v, t)=(v, t+k)$. From the definition of the metric on $\tilde{M}_{A}, \sup _{x} d\left(\Phi_{f}(x), x\right)<$ $\infty$. In fact, the distance from $(v, t)$ to $(v, t+k)$ is exactly $k$. As $\hat{\gamma}$ is an isometry, $\sup _{x} d\left(f(x), \Phi_{f}(x)\right)<\infty$ and so $\sup _{x} d(f(x), x)<\infty$ as well. Thus $f$ satisfies the assumptions in Section 7 .

Let $h: \tilde{M}_{A} \rightarrow \tilde{M}_{A}$ be the homeomorphism given by Lemma 7.5. Since $h$ is a finite distance from the identity, it follows that $\sup _{x} \operatorname{dist}(h(g(x)), \Phi(h(x)))<\infty$. For any leaf $L \in \mathcal{F}^{c s}, h(L)$ is a leaf of $\mathcal{A}^{c s}$ and by Lemma 5.2,

$$
d_{H}(h(g(L)), \Phi(h(L)))<\infty \quad \Rightarrow \quad h(g(L))=\Phi(h(L)) .
$$

The same reasoning applies to $\mathcal{F}^{c u}$ and shows that $h$ is a leaf conjugacy between $g$ and $\Phi$. As $g$ maps $\mathcal{F}^{c s}$ to $\mathcal{F}^{c s}$, it follows from the leaf conjugacy that $\Phi$ maps $\mathcal{A}^{c s}$ to $\mathcal{A}^{c s}$, and therefore $e=1$ in the formula given in Lemma A.3. As all of the maps descend to $M_{A}$, the result is proved.

\section{A.2. Finite quotients of suspension manifolds.}

Proof of Proposition 1.2. Suppose $M$ is finitely covered by $M_{A}$. Without loss of generality (see the discussion at the end of Section 2), assume that this is a normal covering. By Lemma 2.2, an iterate of the partially hyperbolic diffeomorphism $f: M \rightarrow M$ lifts to $g: M_{A} \rightarrow M_{A}$ and, by Proposition [1.3, we may assume that this lift $g$ is homotopic to the identity.

If there is a $g$-periodic torus tangent to $E^{c s}$ or $E^{c u}$ in $M_{A}$, it would quotient to an $f$-periodic torus on $M$ and the results in $\mathrm{RHRHU}_{1}$ would imply that $M$ is a suspension manifold. Therefore, we may freely assume there is no such torus.

Propositions 1.4 and 1.5 imply that $g$ is leaf conjugate to a suspension Anosov flow. As a consequence of Lemma 7.2 , there is a center foliation $\mathcal{F}$ on $M_{A}$ and a map $p: M_{A} \rightarrow$ $\mathbb{R} / \mathbb{Z}$, such that restricted to each leaf of $\mathcal{F}, p$ is a $C^{1}$ covering.

Let $H$ be the finite group of deck transformations $\tau: M_{A} \rightarrow M_{A}$ associated to the covering $M_{A} \rightarrow M$. By Proposition 2.3, $\tau(\mathcal{F})=\mathcal{F}$ for all $\tau \in H$. The space of leaves of $\mathcal{F}$ is a non-Hausdorff space homeomorphic to $\mathbb{T}^{2} / A$. By the Lefschetz fixed point formula, for a homeomorphism $h: \mathbb{T}^{2} \rightarrow \mathbb{T}^{2}$, either $h$ has a fixed point or $A \circ h$ has a fixed point (or both). This implies that a deck transformation $\tau \in H$ fixes at least one leaf $L \in \mathcal{F}$. If $\tau$ reversed the orientation of leaves, it would have a fixed point on $L$, which is impossible for a non-trivial deck transformation. Thus, all $\tau \in H$ preserve the orientation of $\mathcal{F}$. 
Define a map $\hat{p}: M_{A} \rightarrow \mathbb{R} / \mathbb{Z}$ by

$$
\hat{p}(x)=\sum_{\tau \in H} p(\tau(x))
$$

Then, $\hat{p} \circ \tau=\hat{p}$ for all $\tau \in H$, the set $\hat{p}^{-1}(\{0\})$ consists of $n$ disjoint tori where $n=|H|$, and each $\tau$ permutes these tori. Choose one torus and call it $S$. Starting at a point $x \in S$, flow forward along the leaf $\mathcal{F}(x)$ until it intersects a torus of the form $\tau_{1} S$ at a point $y$. Define $\psi(x)=\tau_{1}^{-1}(y)$. This defines a continuous map $\psi: S \rightarrow S$ and the choice of $\tau_{1}$ is independent of $x$.

Define $H_{0}:=\{\tau \in H: \tau(S)=S\}$. Then, $\psi$ quotients to a function $\hat{\psi}: S / H_{0} \rightarrow S / H_{0}$, and one can verify that $M$ is homeomorphic to the manifold defined as the suspension of $\hat{\psi}$. As $\psi^{n}$ is topologically conjugate to a power of $A, \hat{\psi}$ is expansive and therefore topologically conjugate to a hyperbolic toral automorphism $[\mathrm{L}]$.

This shows that $M$ is homeomorphic to a suspension manifold. As these manifolds are three-dimensional, they are diffeomorphic as well [Mo].

\section{A.3. Quotients of the torus and nilmanifolds.}

Proposition A.7. Suppose $f$ is a homeomorphism on $\mathbb{T}^{3}$ such that the induced automorphism on $H_{1}\left(\mathbb{T}^{3}, \mathbb{R}\right)$ is hyperbolic. If $f$ descends to a homeomorphism of a finite quotient $\mathbb{T}^{3} / \Gamma$, then this quotient is homeomorphic to the 3-torus.

Proof. Suppose $\mathbb{T}^{3} / \Gamma$ is such a quotient. Then $\Gamma$ is a finite group of fixed-point free homeomorphisms of $\mathbb{T}^{3}$. As explained in [HJKL], we may freely assume that each $\gamma \in \Gamma$ is affine. That is, $\gamma$ is of the form $x \mapsto A x+b$ where $A$ is a toral automorphism and $b \in \mathbb{T}^{3}$. As $f$ descends to the quotient, the map $\Gamma \rightarrow \Gamma, \gamma \mapsto f \gamma f^{-1}$ is a well-defined automorphism. As $\Gamma$ is finite, there is $n$ such that $\gamma=f^{n} \gamma f^{-n}$ for all $\gamma \in \Gamma$. Assume $n=1$.

The Lefschetz number of a homeomorphism of the 3-torus is

$$
\left(1-\lambda_{1}\right)\left(1-\lambda_{2}\right)\left(1-\lambda_{3}\right)
$$

where the $\lambda_{i}$ are the eigenvalues of the induced automorphism of $H_{1}\left(\mathbb{T}^{3}, \mathbb{R}\right)$ Man. Consider $\gamma \in \Gamma$. As $\gamma$ is fixed-point free, one of these eigenvalues must be equal to one. The associated eigenspace is given by the solution of a system of linear equations with integer coefficients. As this can be solved over $\mathbb{Q}$, the first homology group with coefficients in $\mathbb{Z}$ has a subgroup defined by

$$
E=\left\{v \in H_{1}\left(\mathbb{T}^{3}, \mathbb{Z}\right): \gamma_{*} v=v\right\}
$$

which is non-empty.

Since $f$ and $\gamma$ commute, $f_{*}$ on $H_{1}\left(\mathbb{T}^{3}, \mathbb{Z}\right)$ restricts to an automorphism of $E$. If $E$ is rank 1 , the automorphism shows that $f_{*}$ has an eigenvalue of \pm 1 . If $E$ is rank 2, the 
automorphism shows that $f_{*}$ has two eigenvalues whose product is \pm 1 , and the third eigenvalue is therefore \pm 1 . Neither case is possible if $f_{*}$ is hyperbolic. Hence, $E$ has full rank and by its definition $E=H_{1}\left(\mathbb{T}^{3}, \mathbb{Z}\right)$. Writing $\gamma$ as an affine map $x \mapsto A x+b$, it must be that $A$ is the identity. Thus, $\Gamma$ is a group of translations, and $\mathbb{T}^{3} / \Gamma$ is homeomorphic to the 3 -torus.

Proposition A.8. Suppose $f$ is a homeomorphism on $\mathbb{T}^{3}$ such that the induced automorphism on $H_{1}\left(\mathbb{T}^{3}, \mathbb{R}\right)$ has eigenvalues $\lambda_{1}, \lambda_{2}, \lambda_{3}$ satisfying

$$
0<\left|\lambda_{1}\right|<\left|\lambda_{2}\right|=1<\left|\lambda_{3}\right| .
$$

If $f$ descends to a homeomorphism of a finite quotient $\mathbb{T}^{3} / \Gamma$, then this quotient is either homeomorphic to the 3-torus or double covered by the 3-torus.

Proof. As in the last proof, after replacing $f$ with an iterate, we may freely assume that $f \gamma=\gamma f$ for all $\gamma$ and that each $\gamma$ is an affine map on $\mathbb{T}^{3}$. Also, assume that the eigenvalues $\lambda_{i}$ are all positive.

Define $\Gamma_{0}$ as the set of $\gamma \in \Gamma$ which are translations $x \mapsto x+b$. Then, $\Gamma_{0}$ is a normal subgroup, $f$ descends to $\mathbb{T}^{3} / \Gamma_{0}$ and the quotient is homeomorphic to $\mathbb{T}^{3}$. Therefore, up to replacing $\mathbb{T}^{3}$ by $\mathbb{T}^{3} / \Gamma_{0}$, we may freely assume that no non-trivial $\gamma \in \Gamma$ is a translation. That is, $\gamma_{*} \neq I d$ where $\gamma_{*}$ is the induced automorphism of $H_{1}\left(\mathbb{T}^{3}, \mathbb{Z}\right)$.

Now suppose there is a non-trivial element $\gamma \in \Gamma$. Our goal is to show that $\gamma$ is the unique non-trivial element.

As $\lambda_{2}=1$ is an eigenvalue, arguing as in the last proof, there is $v \in H_{1}\left(\mathbb{T}^{3}, \mathbb{Z}\right)$ such that $f_{*} v=v$. Choosing an appropriate basis for $H_{1}\left(\mathbb{T}^{3}, \mathbb{Z}\right) \cong \mathbb{Z}^{3}$, write $f_{*}$ as a $3 \times 3$ matrix

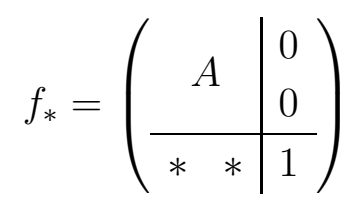

where $A \in G L(2, \mathbb{Z})$ is hyperbolic. As $\gamma_{*}$ commutes with $f_{*}$,

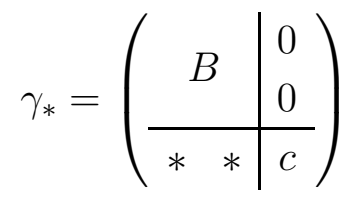

where $A B=B A$. The upper-right entries are zero because that is the only $2 \times 1$ matrix $X$ which satisfies $A X=X$. Since $\Gamma$ is finite, there is $n$ such that $\gamma_{*}^{n}=I d$. Consequently, $c= \pm 1$ and, by Lemma A.2, $B= \pm I d$.

We now consider the four cases for $B$ and $c$.

Case 1. $B=+I d$ and $c=+1$.

Here, $\gamma_{*}$ is a lower triangular matrix with ones on the diagonal and $\gamma_{*}^{n}=I d$ for some 
$n$. This implies that $\gamma_{*}=I d$, which is not possible by an earlier assumption. Therefore, this case cannot occur.

Case 2. $B=-I d$ and $c=-1$.

In this case, $\gamma_{*}$ has -1 as the sole eigenvalue. Using the Lefschetz fixed point theorem as in the previous proof, $\gamma$ would have a fixed point, a contradiction.

Case 3. $B=+I d$ and $c=-1$.

Suppose $\gamma^{\prime}$ is another non-trivial element of $\Gamma$ where the matrix $\gamma_{*}^{\prime}$ has corresponding submatrices $B^{\prime}$ and $c^{\prime}$. If $B^{\prime}=-I d$ and $c^{\prime}=+1$, then $B B^{\prime}=-I d, c c^{\prime}=-1$, and the product $\gamma \gamma^{\prime} \in \Gamma$ would have a matrix of the form already ruled out in case 2 above. Therefore, $B^{\prime}=+I d, c=-1$, and the product $\gamma \gamma^{\prime}$ is of the form considered in case 1. This is only possible if $\gamma \gamma^{\prime}$ is the identity element of $\Gamma$. Since this holds for any non-trivial $\gamma^{\prime} \in \Gamma$, it follows that $\Gamma$ is the two element group $\{I d, \gamma\}$.

Case 4. $B=-I d$ and $c=+1$.

This case is nearly identical to case 3 and is left to the reader.

Not including the torus itself, there are exactly three manifolds (up to either homeomorphism or diffeomorphism) double covered by the 3-torus [LSY]. They are defined by quotienting $\mathbb{T}^{3}=\mathbb{R}^{3} / \mathbb{Z}^{3}$ by one of the following maps

$$
\begin{aligned}
& \tau_{1}(x, y, z)=\left(-x,-y, z+\frac{1}{2}\right) \\
& \tau_{2}(x, y, z)=\left(x+\frac{1}{2}, y,-z\right) \\
& \tau_{3}(x, y, z)=\left(x+z+\frac{1}{2}, y+z,-z\right) .
\end{aligned}
$$

All three resulting manifolds admit partially hyperbolic diffeomorphisms, as demonstrated by the toral automorphisms generated by the matrices

$$
\left(\begin{array}{lll}
2 & 1 & 0 \\
1 & 1 & 0 \\
0 & 0 & 1
\end{array}\right), \quad\left(\begin{array}{lll}
3 & 1 & 0 \\
2 & 1 & 0 \\
0 & 0 & 1
\end{array}\right) \text {, and }\left(\begin{array}{lll}
5 & 2 & 3 \\
2 & 1 & 1 \\
0 & 0 & 1
\end{array}\right) \text {. }
$$

Further, the classification up to leaf conjugacy can be extended from the 3-torus to its finite quotients. To do this, we first state a consequence of the classification on the 3-torus.

Proposition A.9. Suppose $f_{T}, g_{T}: \mathbb{T}^{3} \rightarrow \mathbb{T}^{3}$ are partially hyperbolic, dynamically coherent diffeomorphisms, with lifts $f, g: \mathbb{R}^{3} \rightarrow \mathbb{R}^{3}$ such that the induced automorphisms $f_{*}$ and $g_{*}$ on $\pi_{1}\left(\mathbb{T}^{3}\right)$ are equal. Then, there is a leaf conjugacy $h: \mathbb{R}^{3} \rightarrow \mathbb{R}^{3}$ between $f$ and $g$ which descends to a leaf conjugacy $h_{T}: \mathbb{T}^{3} \rightarrow \mathbb{T}^{3}$ between $f_{T}$ and $g_{T}$ such that $h_{*}$ is the identity.

Further, $h$ is unique up to sliding along center leaves. That is, if $h^{\prime}$ is another such leaf conjugacy, then there is $C>0$ such that for all $x \in \mathbb{R}^{3}, h(x)$ and $h^{\prime}(x)$ lie on the same center leaf and $d_{c}\left(h(x), h^{\prime}(x)\right)<C$. 
Proof. As shown in $\left[\mathrm{H}_{1}\right.$ and extended to the pointwise case in [HP], if $f$ and $g$ have the same action on $\pi_{1}\left(\mathbb{T}^{3}\right)$, they are both leaf conjugate to the same linear map on $\mathbb{R}^{3}$. The stated results then follow from this and the properties proven in $\left[\mathrm{H}_{1}\right]$.

Let $\operatorname{Aff}\left(\mathbb{R}^{n}\right)$ denote the group of affine maps on $\mathbb{R}^{n}$.

Proposition A.10. Suppose that $f_{M}$ is a partially hyperbolic diffeomorphism on a 3manifold $M$ with virtually abelian fundamental group and that there is no 2-torus tangent to $E^{c} \oplus E^{u}$ or $E^{c} \oplus E^{s}$. Then, there is a subgroup $\Gamma<\operatorname{Aff}\left(\mathbb{R}^{3}\right)$, and a partially hyperbolic linear map $A: \mathbb{R}^{3} \rightarrow \mathbb{R}^{3}$ such that $A$ descends to a map $A_{M}: \mathbb{R}^{3} / \Gamma \rightarrow \mathbb{R}^{3} / \Gamma$ which is leaf conjugate to $f_{M}$.

Proof. As $M$ is virtually nilpotent, it is finitely covered by a circle bundle over a 2-torus [Par]. To be virtually abelian, this circle bundle must be trivial. That is, $M$ is finitely covered by the 3 -torus.

If $M$ is diffeomorphic to the 3 -torus, the proof follows from $\mathrm{H}_{1}$ and $[\mathrm{HP}$.

Otherwise, by the results of this section, we may freely assume that $M=\mathbb{R}^{3} / \Gamma$, where $\Gamma$ has an index-two subgroup $\Gamma_{0}$ consisting of translations $x \mapsto x+z$ for $z \in \mathbb{Z}^{3}$. As $\Gamma_{0}$ is the maximal nilpotent subgroup of $\Gamma$, it is preserved by every automorphism. Choose some element of $\Gamma \backslash \Gamma_{0}$ and call it $\alpha$.

Lift $f_{M}$ to $f: \mathbb{R}^{3} \rightarrow \mathbb{R}^{3}$. This defines an automorphism $f_{*}: \Gamma \rightarrow \Gamma$, and by the theorems of Bieberbach (see, for instance, [LR]), there is an affine map $g: \mathbb{R}^{3} \rightarrow \mathbb{R}^{3}$, $g(x)=A x+b$ such that $g$ descends to a diffeomorphism $g_{M}: M \rightarrow M$, and the induced automorphism $g_{*}: \Gamma \rightarrow \Gamma$ is equal to $f_{*}$.

As $f$ is partially hyperbolic, its action on $\Gamma_{0} \cong \mathbb{Z}^{3}$ is partially hyperbolic $([\mathrm{BI}])$. Moreover, by [Pot] (or [HP, Proposition 3.3]), it has eigenvalues $\lambda_{1}, \lambda_{2}, \lambda_{3}$ such that

$$
\left|\lambda_{1}\right|<\left|\lambda_{2}\right|<\left|\lambda_{3}\right| \text { and }\left|\lambda_{1}\right|<1<\left|\lambda_{3}\right|
$$

By Proposition A.7 and the assumption that $M$ is not the 3-torus, it must be that $\left|\lambda_{2}\right|=1$. Then, $g$ is partially hyperbolic and dynamically coherent. As the center bundle of $g$ is uniquely integrable, so is the center bundle of the quotiented map $g_{M}$ on $M$. This shows that the center foliation of $g$ is $\Gamma$-invariant.

Let $h: \mathbb{R}^{3} \rightarrow \mathbb{R}^{3}$ be the leaf conjugacy given by Proposition A.9. That is, $h g(\mathcal{L})=$ $f h(\mathcal{L})$ for every center leaf $\mathcal{L}$ of $g$, and $h \gamma=\gamma h$ for all $\gamma \in \Gamma_{0}$. As $\Gamma_{0}$ is normal, $\gamma \alpha h \alpha^{-1}=\alpha h \alpha^{-1} \gamma$ for all $\gamma \in \Gamma_{0}$. Also, there is $\beta \in \Gamma_{0}$ such that $\alpha f \alpha^{-1}=f \beta$. Since, 
$f_{*}=g_{*}$, it follows that $\alpha g \alpha^{-1}=g \beta$, using the same $\beta$. Then,

$$
\begin{aligned}
f h(\mathcal{L}) & =h g(\mathcal{L}) \Rightarrow \\
\alpha f \alpha^{-1} \alpha h \alpha^{-1}(\mathcal{L}) & =\alpha h \alpha^{-1} \alpha g \alpha^{-1}(\mathcal{L}) \Rightarrow \\
f \beta \alpha h \alpha^{-1}(\mathcal{L}) & =\alpha h \alpha^{-1} g \beta(\mathcal{L}) \Rightarrow \\
f \alpha h \alpha^{-1} \beta(\mathcal{L}) & =\alpha h \alpha^{-1} g \beta(\mathcal{L}) \Rightarrow \\
f \alpha h \alpha^{-1}(\mathcal{L}) & =\alpha h \alpha^{-1} g(\mathcal{L}),
\end{aligned}
$$

so $\alpha h \alpha^{-1}$ is also a leaf conjugacy. By the uniqueness given in Proposition A.9, $h(\mathcal{L})=$ $\alpha h \alpha^{-1}(\mathcal{L})$ for every center leaf $\mathcal{L}$ of $g$. That is, $h$ and $\alpha h \alpha^{-1}$ are c-equivalent, as defined in section 6 of $\left[\mathrm{H}_{1}\right]$. That section explains how to average a finite number of $c$-equivalent leaf conjugacies to get a new leaf conjugacy. If we define $h_{1}$ as such an average of $h$ and $\alpha h \alpha^{-1}$, then one can verify that $h_{1}$ is a leaf conjugacy on $\mathbb{R}^{3}$ which quotients to $M=\mathbb{R}^{3} / \Gamma$.

We have proven that $f_{M}$ is leaf conjugate to a quotient of an affine map $g: \mathbb{R}^{3} \rightarrow$ $\mathbb{R}^{3}, \quad x \mapsto A x+b$, whereas the proposition claims that $f_{M}$ is leaf conjugate to a linear map. By conjugating $g$ with a translation $x \mapsto x+c$ for $c \in \mathbb{R}^{3}$, we can replace $g$ by the map $x \mapsto A x+A c-c+b$. Hence, we can assume that $b$ is in the null space of $A-I d$. This conjugation means that $\Gamma<\operatorname{Aff}\left(\mathbb{R}^{3}\right)$ will also be replaced by a conjugate subgroup of $\operatorname{Aff}\left(\mathbb{R}^{3}\right)$. Then, $(A-I d) b=0$ implies that $b$ and the origin of $\mathbb{R}^{3}$ lie in the same center leaf of $g$. This shows that $g$ is leaf conjugate to the linear map $A$ and this leaf conjugacy (the identity on $\mathbb{R}^{3}$ ) descends to the quotient $\mathbb{R}^{3} / \Gamma$, completing the proof.

Similar results hold for finite quotients of 3-dimensional nilmanifolds. Let $\mathcal{H}$ denote the Heisenberg group, consisting of all matrices of the form

$$
\left(\begin{array}{lll}
1 & x & z \\
0 & 1 & y \\
0 & 0 & 1
\end{array}\right)
$$

under multiplication. For any integer $k \geq 1$, the set of matrices with $x, y \in \mathbb{Z}$ and $z \in \frac{1}{k} \mathbb{Z}$ defines a discrete subgroup $\Gamma_{k}$, and the quotient $\mathcal{H} / \Gamma_{k}$ is a compact manifold, a nilmanifold, denoted by $\mathcal{N}_{k}$. For each $\mathcal{N}_{k}$, the homology group $H_{1}\left(\mathcal{N}_{k}, \mathbb{R}\right)$ is twodimensional. In fact, the projection

$$
\mathcal{H} \rightarrow \mathbb{R}^{2}, \quad\left(\begin{array}{lll}
1 & x & z \\
0 & 1 & y \\
0 & 0 & 1
\end{array}\right) \mapsto(x, y)
$$

defines a projection $\mathcal{N}_{k} \rightarrow \mathbb{T}^{2}$, and this projection induces the isomorphism between $H_{1}\left(\mathcal{N}_{k}, \mathbb{R}\right)$ and $H_{1}\left(\mathbb{T}^{2}, \mathbb{R}\right)$. An affine map on $\mathcal{H}$ is a map of the form $x \mapsto \Phi(x) \cdot c$ where 
$\Phi: \mathcal{H} \rightarrow \mathcal{H}$ is a Lie group automorphism and $c \in \mathcal{H}$. An affine map on $\mathcal{N}_{k}$ is a quotient of an affine map on $\mathcal{H}$.

Proposition A.11. Suppose $f$ is a homeomorphism of $\mathcal{N}_{k}$ such that the induced automorphism on $H_{1}\left(\mathcal{N}_{k}, \mathbb{R}\right)$ is hyperbolic. If $f$ descends to a homeomorphism of a finite quotient $\mathcal{N}_{k} / \Gamma$, then this quotient is either homeomorphic to $\mathcal{N}_{\ell}$ or double covered by $\mathcal{N}_{\ell}$ for some $\ell$.

Proof. As in the proofs of Propositions A.7 and A.8, we may assume the elements of $\Gamma$ are affine maps. (See [LR] and [CS].) As explained in Section 2 of $\mathrm{H}_{2}$, to every homeomorphism of $\mathcal{N}_{k}$ is associated an automorphism of the Lie algebra $\mathfrak{h}$, and this automorphism can be written as a $3 \times 3$ matrix

$$
\left(\begin{array}{c|c}
A & 0 \\
& 0 \\
\hline * \quad * & \operatorname{det}(A)
\end{array}\right)
$$

In fact, $A$ has integer entries and it is the matrix given by the automorphism induced by $f$ on $H_{1}\left(\mathcal{N}_{k}, \mathbb{R}\right)$ (see the discussion after Proposition 5.1 in $\left[\mathrm{H}_{2}\right]$ ).

Using these properties, the proof follows in the same manner as in Proposition A.8.

The finite quotients of 3-nilmanifolds have been classified [DIKL]. If $M$ is double covered by $\mathcal{N}_{k}$, but is not itself a nilmanifold, then $k$ is even and $M$ is diffeomorphic to $\mathcal{H} /\left\langle\Gamma_{k}, \tau_{k}\right\rangle$ where

$$
\tau_{k}: \mathcal{H} \rightarrow \mathcal{H}, \quad\left(\begin{array}{ccc}
1 & x & z \\
0 & 1 & y \\
0 & 0 & 1
\end{array}\right) \mapsto\left(\begin{array}{ccc}
1 & -x & z+\frac{1}{2 k} \\
0 & 1 & -y \\
0 & 0 & 1
\end{array}\right)
$$

To see this, look at the list of quotients given in [DIKL] and note that only item 2 of the list is a double cover.

Every such manifold supports a partially hyperbolic diffeomorphism, as evidenced by the map

$$
\mathcal{H} \rightarrow \mathcal{H}, \quad\left(\begin{array}{lll}
1 & x & z \\
0 & 1 & y \\
0 & 0 & 1
\end{array}\right) \mapsto\left(\begin{array}{ccc}
1 & 5 x+2 y & z+5 x^{2}+y^{2}+4 x y \\
0 & 1 & 2 y+z \\
0 & 0 & 1
\end{array}\right)
$$

This map also shows that $\mathcal{N}_{k}$ supports a partially hyperbolic diffeomorphism for every $k$ (even or odd).

Proposition A.12. Suppose $f_{M}$ is a partially hyperbolic diffeomorphism on a 3-manifold $M$ with a fundamental group which is virtually nilpotent and not virtually abelian. Then, there is a subgroup $\Gamma<\operatorname{Aff}(\mathcal{H})$, and a partially hyperbolic automorphism $\Phi: \mathcal{H} \rightarrow \mathcal{H}$ such that $\Phi$ descends to a map $\Phi_{M}: \mathcal{H} / \Gamma \rightarrow \mathcal{H} / \Gamma$ which is leaf conjugate to $f_{M}$. 
Proof. As shown in [Par], $M$ is finitely covered by a circle bundle over the torus. As the fundamental group is not virtually abelian, the circle bundle is non-trivial and such nontrivial circle bundles are exactly the nilmanifolds $\mathcal{N}_{k}$. Then, $f_{M}$ is dynamically coherent by [HP, Theorem 1.2].

The proof in this case now follows almost exactly as the proof of Proposition A.10, but with $\mathbb{R}^{3}$ replaced by $\mathcal{H}$. The relevant theorem of Bieberbach used to construct an affine map $g$ also holds in the case of nilmanifolds, as proven by Lee and Raymond [LR]. Partial hyperbolicity of $g$ with $\left|\lambda_{1}\right|<\left|\lambda_{2}\right|=1<\left|\lambda_{3}\right|$ is proven in $\left[\mathrm{H}_{2}\right]$.

\section{ApPendix B. On CENTER-STABLE TORI}

This appendix treats partially hyperbolic diffeomorphisms admitting center-stable or center-unstable tori. The first subsection proves Proposition 3.6. In the second subsection the case of absolute partial hyperbolicity is discussed: it is shown that under this more restrictive definition, the existence of such tori is not possible. This allows to recover the main result of $\mathrm{BBI}_{2}$ without (explicit) use of quasi-isometry and extend it to the case of suspension manifolds.

\section{B.1. Proof of Proposition 3.6 .}

Proof of Proposition 3.6. Assume that $\mathcal{F}^{c s}$ has a leaf $L$ which projects down to a compact surface $T$ in $M$. As $T$ is tangent to $E^{c s}$ it admits a foliation without circle leaves and thus must be a torus. Since $L$ is homeomorphic to a plane, the torus $T$ is incompressible. If $L_{1}$ and $L_{2}$ are leaves of $\mathcal{F}^{c s}$ which project to tori, item (5) of Theorem 3.1 shows their projections are disjoint modulo isotopy. By classical results in 3-manifold topology, there are finitely many disjoint incompressible tori modulo isotopy, so by replacing the diffeomorphism $f$ on $M$ by an iterate, assume $f(T)$ is isotopic to $T$.

Take $x \in T$ and consider a sequence $n_{k}$ such that $x_{k}=f^{-n_{k}}(x)$ converges. Lift $x_{k}$ to a convergent sequence $\tilde{x}_{k}$ in the cover $\tilde{M}$ and lift each torus $T_{k}=f^{-n_{k}}(T)$ to a leaf $L_{k}$ in $\mathcal{F}^{c s}$ through $\tilde{x}_{k}$. As shown by [BI, Lemma 7.1], these leaves $L_{k}$ converge in the $C^{1}$ topology to a leaf $L_{\infty}$ in $\mathcal{F}^{c s}$. Since there is a subgroup $\Gamma$ isomorphic to $\mathbb{Z}^{2}$ of deck transformations which fix all the leaves $L_{k}$ this group $\Gamma$ also fixes $L_{\infty}$.

Projecting down, the limit leaf $T_{\infty} \subset M$ contains a copy of $\mathbb{Z}^{2}$ in its fundamental group and is therefore compact $([\mathrm{Ri}])$. Arguing as above, $T_{\infty}$ is a torus. Construct a normal neighborhood $N$ of $T_{\infty}$ consisting of small unstable segments. Then, there is an arbitrarily large iterate $f^{-\ell}$ which maps a torus $T_{k_{1}}$ to $T_{k_{2}}$ where both tori are arbitrarily close to $T_{\infty}$. Since these tori are transverse to the unstable bundle on $M$, one can show that $f^{-\ell}(\bar{N}) \subset N$ and therefore there is a normally repelling $f$-periodic torus tangent to $E^{c s}$ inside $N$. 
Corollary B.1. Assume $f: M \rightarrow M$ is a partially hyperbolic diffeomorphism such that the bundles $E^{s}, E^{c}, E^{u}$ are orientable then $M$ admits a codimension one foliation without compact leaves.

Proof. If $M$ admits an invariant torus tangent to $E^{c s}$ or $E^{c u}$, then it is one of the manifolds listed in [RHRU 1 , all of which have admit foliations without compact leaves. Otherwise, the result follows by Propositions 3.5 and 3.6.

B.2. Absolute partial hyperbolicity and center-stable tori. To end this appendix, we give a different proof of the main result of $\mathrm{BBI}_{2}$, which also applies to solvmanifolds. A partially hyperbolic diffeomorphism $f: M \rightarrow M$ with splitting $T M=E^{s} \oplus E^{c} \oplus E^{u}$ is absolutely partially hyperbolic if there exist constants $0<\gamma_{1}<1<\gamma_{2}$ and $N>0$ such that for every $x \in M$ :

$$
\left\|\left.D f^{N}\right|_{E^{s}(x)}\right\|<\gamma_{1}<\left\|\left.D f^{N}\right|_{E^{c}(x)}\right\|<\gamma_{2}<\left\|\left.D f^{N}\right|_{E^{u}(x)}\right\| .
$$

Theorem B.2. Let $f: M \rightarrow M$ be a partially hyperbolic diffeomorphism admitting a two dimensional $f$-periodic torus $T$ tangent to $E^{c s}$. Then, $f$ is not absolutely partially hyperbolic.

Proof. Assume $T^{c s}$ is an $f$-invariant torus tangent to $E^{c s}$. Then, the dynamics in $T^{c s}$ must be semiconjugated to a certain linear Anosov diffeomorphism $A$ of $\mathbb{T}^{2}$ (see RHRHU 1 ). The entropy of $\left.f\right|_{T^{c s}}$ is at least as big as the entropy of $A$. Using the variational principle and Ruelle's inequality (see $[\mathrm{M}]$ ), for every $\varepsilon>0$ there is an ergodic measure $\mu^{\varepsilon}$ such that the center Lyapunov exponent of $\mu^{\varepsilon}$ is at least $h_{\text {top }}(A)-\varepsilon$.

On the other hand, adapting the proof of Lemma 4.5 in $\left[\mathrm{H}_{2}\right]$ shows that the asymptotic rate of expansion, $\gamma_{2}$, along the center is strictly smaller than than $h_{t o p}(A)$, which is equal to the largest Lyapunov exponent of $A$. This gives a contradiction.

As a consequence we obtain that every absolutely partially hyperbolic diffeomorphism of a 3-manifold with (virtually) solvable fundamental group is dynamically coherent.

\section{REFERENCES}

[BR] M. Baake and J. A. G. Roberts, Reversing symmetry groups of $G l(2, \mathbb{Z})$ and $P G l(2, \mathbb{Z})$ matrices with connections to cat maps and trace maps. J. Phys. A: Math. Gen. 30 (1997) 1549-1573.

[BDV] C. Bonatti, L. Diaz and M. Viana, Dynamics Beyond Uniform Hyperbolicity. A global geometric and probabilistic perspective, Encyclopaedia of Mathematical Sciences 102. Mathematical Physics III. Springer-Verlag (2005).

[BoW] C. Bonatti and A. Wilkinson, Transitive partially hyperbolic diffeomorphisms on 3-manifolds, Topology 44 (2005) 475-508.

[BBI] M. Brin, D. Burago and S. Ivanov, On partially hyperbolic diffeomorphisms of 3-manifolds with commutative fundamental group. Modern dynamical systems and applications Cambridge Univ. Press, Cambridge, (2004) 307-312. 
$\left[\mathrm{BBI}_{2}\right]$ M. Brin, D. Burago and S. Ivanov, Dynamical coherence of partially hyperbolic diffeomorphisms of the 3-torus. Journal of Modern Dynamics 3 (2009) 1-11.

[Bru] M. Brunella, Expansive flows on Seifert Manifolds and on Torus bundles, Bull. Braz. Math. Soc 241 (1993) 89-104.

[BI] D. Burago and S. Ivanov, Partially hyperbolic diffeomorphisms of 3-manifolds with abelian fundamental groups. Journal of Modern Dynamics 2 (2008) 541-580.

$[\mathrm{BuW}] \mathrm{K}$. Burns and A. Wilkinson, Dynamical coherence and center bunching, Discrete and Continuous Dynamical Systems A (Pesin birthday issue) 22 (2008) 89-100.

[CC] A. Candel and L. Conlon, Foliations I and II, Graduate studies in Mathematics 60 American Math. Society (2003).

[CS] D. Choi and J. Shin, Free actions on finite abelian groups on 3-dimensional nilmanifolds, J. Korean Math. Soc. 42 (2005) 795-826.

[DIKL] K. Dekimpe, P. Igodt, S. Kim and K.B. Lee, Affine structures for closed 3-dimensional manifolds with nil-geometry, Quart. J. Math. Oxford Ser. 46 (1995) 141-167.

[Fu] F. Brock Fuller, On the surface of section and periodic trajectories, Amer. J. Math., 87 (1965) 473-480.

[GS] É. Ghys and V. Sergiescu, Stabilite et conjugaison differentiable pour certains feuilletages, Topology 19 (1980) 179-197.

[HJKL] K.Y. Ha, J.H. Jo, S. W. Kim and J.B. Lee, Classification of free actions of finite groups on the 3-torus, Topology and its applications 121 (2002) 469-507.

$\left[\mathrm{H}_{1}\right]$ A. Hammerlindl, Leaf conjugacies in the torus, Ergodic theory and dynamical systems 33 Issue 03 (2013), pp $896-933$.

$\left[\mathrm{H}_{2}\right]$ A. Hammerlindl, Partial hyperbolicity on 3-dimensional nilmanifolds, Discrete and Continuous Dynamical Systems A, 33:8 (2013) 3641-3669.

[HP] A. Hammerlindl and R. Potrie, Pointwise partial hyperbolicity in 3-dimensional nilmanifolds, Journal of the London Math. Soc. 89 (3) (2014) : 853-875.

[HPS] M. Hirsch, C. Pugh and M. Shub, Invariant Manifolds, Springer Lecture Notes in Math., $\mathbf{5 8 3}$ (1977).

[LR] K.B. Lee and F. Raymond, Rigidity of almost crystallographic groups, In Combinatorial methods in topology and algebraic geometry (Rochester, N.Y. 1982), volume 44 of Contemp. Math. pages 73-78. Amer. Math. Soc. Providence, RI, 1985.

[LSY] K.B. Lee, J. Shin and S. Yokura, Free actions on finite abelian groups on the 3-torus, Topology and its applications 53 (1993) 153-175.

[L] J. Lewowicz, Expansive homeomorphisms on surfaces, Bol. Braz. Mat. Soc. 20 (1989), 113-133.

[LS] R.C. Lyndon, P.E. Schupp, Combinatorial group theory, Ergebnisse der Mathematik und ihrer Grenzgebiete, Band 89. Springer-Verlag, Berlin-New York, (1977). xiv+339 pp.

[Man] A. Manning, There are no new Anosov diffeomorphisms on tori, Amer. J. Math 96 (1974) 422442.

[M] R. Mañe, Teoria Ergódica,Projeto Euclides, CNPq IMPA (1983).

[Mo] E.E. Moise, Affine structures in 3-manifolds v. the triangulation theorem and hauptvermutung, Annals of Math. 56 (1952) 96-114.

[Par] K. Parwani, On 3-manifolds that support partially hyperbolic diffeomorphisms, Nonlinearity 23 (2010) 589-606.

[Pl] J.F. Plante, Foliations of 3-manifolds with solvable fundamental group, Inventiones Math. 51 (1979) 219-230. 
[Pot] R. Potrie, Partial hyperbolicity and foliations in $\mathbb{T}^{3}$, Preprint (2012) arXiv:1206.2860 To appear in Journal of Modern Dynamics.

[Ri] I. Richards, On the classification of non-compact surfaces, Transactions of the A.M.S 1062 (1963) 259-269.

[RHRHU $\left.{ }_{1}\right]$ M.A. Rodriguez Hertz, F. Rodriguez Hertz and R. Ures, Tori with hyperbolic dynamics in 3-manifolds, Journal of Modern Dynamics, 51, (2011) 185-202.

$\left[\mathrm{RHRHU}_{2}\right]$ M. A. Rodriguez Hertz, F. Rodriguez Hertz and R. Ures, A non dynamically coherent example in $\mathbb{T}^{3}$, Preprint, arXiv:1409.0738,

[Wi] A. Wilkinson, Conservative partially hyperbolic dynamics, 2010 ICM Proceedings (2010).

School of Mathematical Sciences, Monash University, Victoria 3800 Australia

E-mail address: andy.hammerlindl@monash.edu

CMat, Facultad de Ciencias, Universidad de la República, Uruguay

$U R L$ : www . cmat.edu. uy/ rpotrie

E-mail address: rpotrie@cmat.edu.uy 